\title{
A Survey on Feature Drift Adaptation: Definition, Benchmark, Challenges and Future Directions
}

\author{
Jean Paul Barddal, Heitor Murilo Gomes, Fabrício Enembreck \\ Graduate Program in Informatics (PPGIa) - Pontifícia Universidade Católica do Paraná \\ Bernhard Pfahringer \\ Department of Computer Science - University of Waikato
}

\begin{abstract}
Data stream mining is a fast growing research topic due to the ubiquity of data in several real-world problems. Given their ephemeral nature, data stream sources are expected to undergo changes in data distribution, a phenomenon called concept drift. This paper focuses on one specific type of drift that has not yet been thoroughly studied, namely feature drift. Feature drift occurs whenever a subset of features becomes, or ceases to be, relevant to the learning task, thus, learners must detect and adapt to these changes accordingly. We survey existing work on feature drift adaptation in both explicit and implicit approaches. Additionally, we benchmark several algorithms and a naive proposal in synthetic and real-world datasets. The results from our experiments indicate the need for future research in this area as even naive approaches produced gains in accuracy while reducing resources usage. Finally, we state current research topics, challenges and future directions for feature drift adaptation.

Keywords: Feature Drift, Feature Selection, Data Stream Mining
\end{abstract}

Email addresses: \{jean.barddal, hmgomes, fabricio\}@ppgia.pucpr.br (Jean Paul Barddal, Heitor Murilo Gomes, Fabrício Enembreck), bernhard@cs.waikato.ac.nz (Bernhard Pfahringer)

Preprint submitted to Journal of Systems and Software

July 25, 2017 


\section{Introduction}

In the last decades the interest in mining massive and potentially unbounded datasets that arrive at rapid rates, namely data streams, has grown substantially. Examples of data streams include, but are not limited to, sensor networks, wearable sensors, computer network traffic sniffers and video surveillance. Aiming at extracting useful knowledge from these massive amounts of data, a variety of inductive learning techniques were developed and achieved concrete results in both supervised $[1,2,3]$ and unsupervised $[4,5,6,7]$ settings.

The most common task in streaming scenarios is classification. In this task, instances are labeled according to a finite set of labels and the goal is to derive a model that accurately classifies upcoming unlabeled instances. Data stream classification algorithms are presented with a great and possibly unbounded amount of data, which are made available to the algorithm in a serialized fastpaced fashion [8]. Moreover, due to the inherent ephemeral aspects of data streams, one must assume that the underlying concept is unstable, i.e. changes in data distribution are expected to occur, a phenomenon called concept drift $[9]$.

Although current techniques for data stream classification handle most of the challenges posed by streaming environments, not much attention has been given to possible changes in the relevance of features through time, a phenomenon called feature drift [10]. To maintain an accurate predictive model on a data stream that exhibits feature drifts, a classifier must be trained and updated on the set of features that is currently relevant. One way to select the relevant subset of features is through feature selection. However, performing feature selection over data streams is still an open research topic since the majority of existing feature selection algorithms require multiple passes over data.

As in batch learning, if an algorithm is capable of discerning between relevant, irrelevant and redundant features, it is expected to compute faster, show lower memory usage (due to diminished dimensionality) and even produce higher prediction accuracy [11]. Nevertheless, performing feature selection incremen- 
tally and adaptively as the stream progresses is not straightforward.

In this paper we review the classification task for data streams (Sec. 2) and the basic concepts of feature selection (Sec. 3). Later, we discuss existing works that perform feature drift adaptation in both explicit and implicit fashions, showing their major limitations (Sec. 4). Several surveyed algorithms are then benchmarked on both synthetic and real datasets that contain feature drifts. We also propose and evaluate a naive algorithm to handle feature drifts. Results obtained corroborate the need for future research on feature selection for data streams (Sec. 5). Finally, we state the challenges of this research area and future directions (Secs. 6 and 7).

\section{Data Stream Mining}

Data acquisition and storage is getting cheaper and easier every day. Recent studies show that 2.5 quintillion of bytes are produced every day, and out of that it is estimated that approximately $90 \%$ of overall stored data were created between 2012 and 2014 [12]. Since it might be difficult to extract useful knowledge from this abundant data, data mining techniques have been widely used for this task $[13,14,8,15]$.

Nowadays, a variety of computational systems create enormous amounts of data, mostly in sequential fashion, and impose several constraints on available processing time and memory space. Extracting interesting patterns from data streams has received growing attention of the data mining community in the last few years $[2,16,4,17]$.

\subsection{Data Stream Classification}

The most common approach for extracting useful knowledge from data streams is classification. Classification is the task that distributes a set of instances into classes (discrete values) accordingly to relations or affinities. Assuming a set of possible classes $Y=\left\{y_{1}, \ldots, y_{c}\right\}$, a classifier builds a model that predicts for every unlabeled instance $\vec{x}_{i}$ its corresponding class $y_{i}$, ideally with close to perfect accuracy [14]. 
The classification task can be formalized as follows: a set of $n$ training instances in the form $\left(\vec{x}_{i}, y_{i}\right)$ where $y_{i}$ is a discrete class label and $\vec{x}_{i}$ is a $d$ dimensional vector of attributes belonging to a feature set $\mathcal{D}$ with cardinality (or number of dimensions) $d$, where the feature set can be categorical, ordinal, numeric or most likely a mix of all three types. A classifier uses the training set to produce a model $f: \vec{x} \rightarrow Y$ that is used to classify future unlabeled instances.

According to the Bayesian theory, classification can be posed as a function of the prior probabilities of the classes $P[y]$ and the class conditional probability density functions ( $p d f s) P[\vec{x} \mid y]$ for all possible classes $y_{i} \in Y$ [8]. Classification decision (labeling) is performed accordingly to the posterior probabilities of the classes, where Eq. 1 states the posterior probability for an arbitrary class $y_{i}$ and $P[\vec{x}]=\sum_{y_{i} \in Y} P\left[y_{i}\right] \times P\left[\vec{x} \mid y_{i}\right]$.

$$
P\left[y_{i} \mid \vec{x}\right]=\frac{P\left[y_{i}\right] \times P\left[\vec{x} \mid y_{i}\right]}{P[\vec{x}]}
$$

Data stream classification, or online classification, is a variant of the machine learning task batch classification. Although both are concerned with the problem of learning a model which is able to predict a nominal value for future unlabeled instances, the difference between these two approaches concerns about how data is presented to the learner. In a batch configuration, a static and entirely accessible dataset is provided to the learning algorithm, which usually performs multiple passes over the training set to build its predictive model. Conversely, in streaming environments, instances are not readily available to the classifier for training, instead, these are presented sequentially over time, and the learner must incrementally adapt its model $f$ as new instances become available [18].

Formally, let $\mathcal{S}=\left[i^{t}\right]_{t=0}^{\infty}$ define a data stream providing instances $i^{t}=$ $\left(\vec{x}^{t}, y^{t}\right)$, each of which arriving at a timestamp $t$, where $\vec{x}^{t}$ is a $d$-dimensional feature vector belonging to a feature set $\mathcal{D}$, and $y_{t}$ is the instance's ground-truth label. 


\subsection{Concept Drift}

Batch learning techniques assume that there is a static dataset generated by a unknown and stationary probability distribution, where the data can be physically stored and analyzed in multiple steps by a batch algorithm. Nonetheless, none of the latter assumptions can be verified in streaming scenarios and the development of data stream classifiers must account for several constraints $[19,8,20]$.

Firstly, instances arrive continuously over time and there is no control over the order that they arrive in nor how they should be processed. Additionally, streams are potentially unbounded, therefore, instances should be discarded right after their processing (or accordingly to available main memory space).

Due to the inherent temporal aspect of data streams, their underlying data distribution is expected to change over time, implying changes in the concept to be learned, a phenomenon called concept drift.

Eq. 2 defines a concept $C$ as a set of prior probabilities of the classes and class-conditional probability density functions [21].

$$
C=\bigcup_{y_{i} \in Y}\left\{\left(P\left[y_{i}\right], P\left[\vec{x} \mid y_{i}\right]\right)\right\}
$$

Given a stream $\mathcal{S}$, retrieved instances $i_{t}$ will be generated by a concept $C_{t}$. If during every instant $t_{i}$ of the stream $C_{t_{i}}=C_{t_{i-1}}$ holds, then $C$ is a stable concept. Otherwise, if between any two timestamps $t_{i}$ and $t_{j}=t_{i}+\Delta$ (with $\Delta \geq 1) C_{t_{i}} \neq C_{t_{j}}$ betides, then a concept drift has occurred.

For more details on the problem of concept drift, the reader is referred to more specific works $[18,8,14]$.

\section{Feature Selection}

Datasets (or streams) for analysis may contain hundreds (or even thousands or millions) of features (attributes), many of which are possibly irrelevant or redundant to the learning task. Dealing with this massive amount of features is not only computationally expensive but it also jeopardizes inductive learning 
algorithms since the training set would cover a dwindling part of the feature space. Even if we assume a large dataset with trillions of uniformly distributed instances in a moderate attribute space of 100 features, only about $10^{-18}$ of the potential space would be covered [22]. Also, high dimensional spaces can be a problem due to the "curse of dimensionality", where learning algorithms based on distance computations are known to fail [17]. To overcome these problems, a variety of feature selection algorithms were developed and aim at performing dimensionality reduction in batch learning $[23,24,11]$.

In the following section we describe important concepts related to feature selection which enable us to later formalize and discuss feature drifts properly.

\subsection{Definitions}

Up to this point, the term "relevance" was used without a formal definition. In this section, we define the concept of relevance in the feature selection task. As stated in $[25,26]$, there are different definitions available in the literature, nevertheless, several may be contradictory and misleading. In this paper we provide the most common definitions by dividing features in three types: relevant, irrelevant and redundant.

Definition 3.1. Assuming $S_{i}=\mathcal{D} \backslash\left\{D_{i}\right\}$, a feature $D_{i}$ is relevant iff

$$
\exists S_{i}^{\prime} \subset S_{i} \text {, such that } P\left[Y \mid D_{i}, S_{i}^{\prime}\right] \neq P\left[Y \mid S_{i}^{\prime}\right] \text { holds }
$$

Otherwise, $D_{i}$ is said to be irrelevant.

According to this definition, if a feature that is statistically relevant, is removed from a feature set, then this will reduce overall prediction power. This definition encompasses two possibilities for a feature to be statistically significant: (i) it is strongly correlated with the class; or (ii) it forms a feature subset with other features, and this subset is strongly correlated with the class [27].

Another aim of feature selection algorithms is to tackle redundant data. A feature becomes redundant due to the existence of other relevant features, which provide similar prediction power. 
Definition 3.2. Assuming $S_{i}=\mathcal{D} \backslash\left\{D_{i}\right\}$, a feature $D_{i}$ is redundant iff

$$
\exists S_{i}^{\prime} \subset S_{i} \text {, such that } P\left[Y \mid D_{i}, S_{i}\right]=P\left[Y \mid S_{i}\right] \wedge P\left[Y \mid S_{i}\right] \neq P\left[Y \mid S_{i}^{\prime}\right]
$$

Several studies proposed the removal of redundant features as this might jeopardize prediction accuracy due to overfitting [28], while others noticed that the removal of this type of feature may cause the exclusion of potentially relevant features [29]. Most of existing works propose to find redundant features through correlations $[30,31,28]$ or clustering similar patterns into feature clusters [32, $33]$.

\subsection{Feature Selection Task Definition}

The feature selection task for data streams is to obtain the optimal subset $\mathcal{D}^{*} \subseteq \mathcal{D}$ of features that represents the concept to be learned from a dataset or data stream. The goal of feature selection is to remove irrelevant and redundant attributes, while maintaining the probability distribution of the original data classes $P[Y]$. Mining this reduced dimensionality dataset implies a smaller number of parameters in the patterns to be discovered, which leads to easier concepts to understand and which provides as good or better accuracy in the predictive model, whilst requiring less data [34].

The problem of feature selection can be formalized as an optimization problem.

Definition 3.3. Assuming the full and variable set of features $\mathcal{D}$, the goal is to select a subset $\mathcal{D}^{*}$ that retains only the relevant information in $\mathcal{S}$. Suppose that the goodness of a subset of features $\mathcal{D}^{\prime} \subseteq \mathcal{D}$ is given by $Q(\cdot)$, then feature selection can be stated as in Eq. 5, where $d_{\max }$ is the upper bound on the number of selected features.

$$
\mathcal{D}^{*}=\underset{\mathcal{D}^{\prime} \subseteq \mathcal{D}}{\operatorname{argmax}} Q\left(\mathcal{D}^{\prime}\right) \text { subject to }\left|\mathcal{D}^{\prime}\right| \leq d_{\max }
$$

Finding $\mathcal{D}^{*}$ is a difficult task that, assuming $d_{\max }=d$, requires an exploratory search which is by definition $\mathcal{O}\left(2^{d}\right)$. In Fig. 1 we present a graphical representation of the features subset space assuming $\mathcal{D}=\left\{D_{1}, D_{2}, D_{3}, D_{4}\right\}$. 


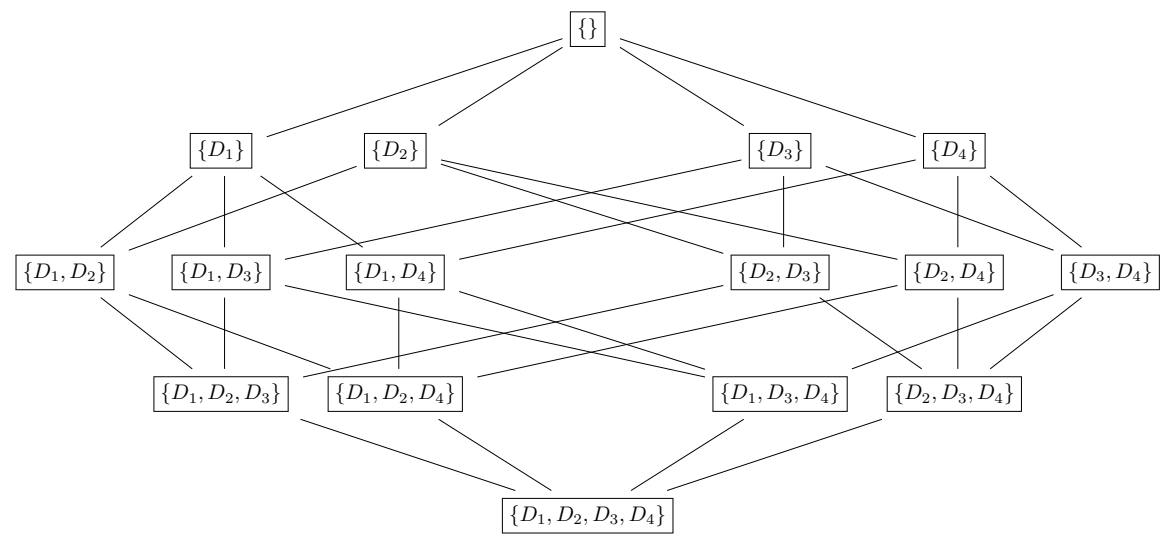

Figure 1: Feature subsets space assuming $\mathcal{D}=\left\{D_{1}, D_{2}, D_{3}, D_{4}\right\}$.

Due to the exponential computational complexity, most algorithms employ some kind of heuristic to guide the selection process, which may lead to suboptimal selected discriminant feature subsets.

\subsection{Taxonomy of Feature Selection Methods}

Existing works on feature selection are commonly divided into three classes: filters, wrappers and embedded methods [35]. In this section we briefly describe these three categories.

Filters. Filters apply statistical measures to assign a score to each feature. Features are then ranked by scores and either selected to be kept or removed given a threshold. These methods are usually univariate and consider each feature independently. Two important traits of filters are their independence from the learning algorithm adopted and low computational cost. Examples of filter methods include the $\chi^{2}$ test, Information Gain, Entropy, Correlation Coefficient Scores, Las Vegas Filters, Relief and ReliefF [11].

Wrappers. Wrappers consider the selection of a subset of features as a search problem, where different combinations are prepared, evaluated and pairwisely compared, usually in bottom-top or top-bottom approaches. A predictive model is used to evaluate each combination of features and to assign a score based on 
prediction accuracy. Therefore, wrappers are sensitive to the learning algorithm's bias, i.e. recognize that certain algorithms may work better with different features [36]. An important drawback of wrappers is their computational cost, which is prohibitive in high dimensional or in real-time scenarios. The most common search processes are: best-first search, stochastic hill-climbing algorithms, forward and backward passes, beam search and simulated annealing.

Embedded methods. Embedded methods learn which features best contribute to the overall accuracy of the learning algorithm while the model is created. Decision tree learning, for example, can be considered to be an embedded method since the construction of the tree and the selection of the features are interleaved, but the selection of features itself is done by filters. Embedded approaches interact directly with the learning algorithm and present better computational complexity than wrappers [37].

\subsection{Feature Drift}

Most existing algorithms for data streams tackle the infinite length and drifting concept characteristics. However, not much attention has been given to feature drifts. Feature drifts occur whenever a subset of features becomes, or ceases to be, relevant to the concept to be learned. This forces a learner to adapt its predictive model to ignore irrelevant attributes and account for the newly relevant ones [21].

Definition 3.4. Given a feature space $\mathcal{D}$ at a timestamp $t$, it is possible to select the ground-truth relevant subset $\mathcal{D}_{t}^{*} \subseteq \mathcal{D}$ such that $\forall D_{i} \in \mathcal{D}_{t}^{*}$ Def. 3.1 holds and $\forall D_{j} \in \mathcal{D} \backslash \mathcal{D}_{t}^{*}$ the same definition does not. A feature drift occurs if, at any two time instants $t_{i}$ and $t_{j}=t_{i}+\Delta, \mathcal{D}_{t_{i}}^{*} \neq \mathcal{D}_{t_{j}}^{*}$ holds.

Definition 3.5. Let $r\left(D_{i}, t_{j}\right) \in\{0,1\}$ denote a function that determines whether Def. 3.1 holds for a feature $D_{i}$ at a timestamp $t_{j}$ of the stream. A positive relevance, i.e. $r\left(D_{i}, t_{i}\right)=1$, states that $D_{i} \in \mathcal{D}^{*}$ at a timestamp $t_{i}$ and that $D_{i}$ impacts the underlying probabilities $P\left[\vec{x} \mid y_{i}\right]$ of the concept $C_{t}$ of $\mathcal{S}$. A feature 
drift occurs whenever the relevance of an attribute $D_{i}$ changes in a timespan between $t_{j}$ and $t_{k}$, as stated in Eq. 6 .

$$
\exists t_{j} \exists t_{k}, t_{j}<t_{k}, r\left(D_{i}, t_{j}\right) \neq r\left(D_{i}, t_{k}\right)
$$

Changes in $r(\cdot, \cdot)$ directly affect the ground-truth decision boundary to be learned by the learning algorithm. Therefore, feature drifts can be posed as a specific type of concept drift that may occur with or without changes in the data distribution $P[\vec{x}][38,10]$.

As in conventional concept drifts, changes in $r(\cdot, \cdot)$ may occur during the processing of the stream. Thus, data stream learners are expected to detect changes in $\mathcal{D}^{*}$, discerning between features that became irrelevant and those that are now relevant and vice-versa. Finally, it is necessary to either (i) discard and learn an entirely new classification model; or (ii) adapt the current model to these drifts [21].

Although feature drifts may occur in a variety of environments, one of the most common domains in which it happens is text mining. In order to exemplify a feature drift, we refer to the e-mail spam detection system presented in [39]. This system was a result of a text mining process on an online news dissemination system. Essentially, this work intended to create an incremental filtering of emails that classifies emails as spam or ham and, based on this classification, decides whether this email is relevant for dissemination among users. The dataset contains 9,324 instances and 39,917 features, such that each attribute represents the presence of a single word (feature) in an instance (e-mail). This dataset, called Spam Corpus, is known for containing a feature drift which occurs gradually around the instance of number $1,500[1,39,10]$ and that highly impacts on the learner.

In Fig. 2a we present a plot of the information gain [40] of two specific attributes presented in this problem, namely "directed" and "info", where one can see that the importance of these two features starts exchanging gradually around instance 1,500 [38]. Detecting and discerning the two features that exchange relevance as the stream progresses is an important task that must be 


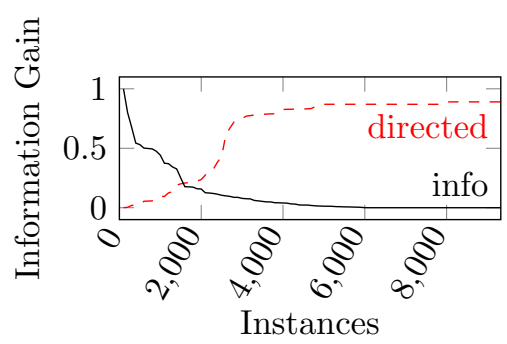

(a) Information gain of features "directed" and "info" during the stream.

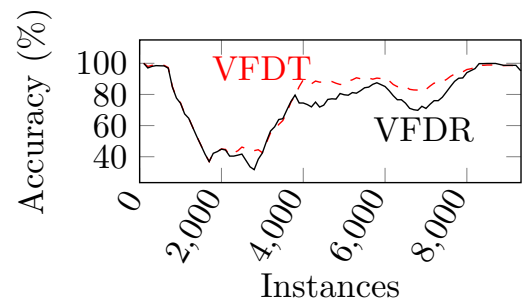

(b) Accuracy obtained for a decision tree (VFDT) and a decision rule learner (VFDR).

Figure 2: Analysis of information gain for two specific features and accuracy obtained on the Spam Corpus dataset. Adapted from [10, 38].

embedded within streaming learning algorithms, since changes greatly impact the accuracy of the model (Fig. 2b) and learning with a subset of the whole feature set is also computationally faster. We refrain from providing a detailed description of these classifiers since the Very Fast Decision Tree (VFDT) and Very Fast Decision Rules (VFDR) are discussed in Secs. 4.1 and 4.2, respectively.

\subsection{A Note on Dynamic Feature Selection versus Streaming Feature Selection}

It is important to emphasize the difference between Dynamic Feature Selection for data streams and Streaming Feature Selection (also commonly referred as Online Feature Selection [23, 24]). Streaming feature selection regards the possibility of finding the best subset of features in a very high-dimensional space (hundreds of thousands or millions of dimensions), which is a typical problem of big data [23]. Although both tasks' objectives overlap, streaming feature selection receives as input a stream of features (not instances), and their inclusion in the model is performed sequentially, without observing future features [41]. Therefore, Streaming Feature Selection is used in batch learning, when the amount of features is gigantic, scaling up to thousands or millions of attributes and the amount of instances is static and does not vary with time. 


\section{Existing Works on Overcoming Feature Drifts}

There are few works in the literature that perform feature selection during stream learning. There are even fewer that aim at explicitly detecting and adapting to feature drifts. In this section we summarize existing algorithms that perform feature selection as the stream progresses, either assuming the existence, or not, of feature drifts.

In Tab. 1 we summarize existing algorithms. We categorize them accordingly to four characteristics: their learning approach, the specific feature selection algorithm, the specific feature drift adaptation method adopted; and whether they perform explicit dynamic feature selection or not.

We start by discussing two important and widely used approaches for classifying data streams: decision trees (Sec. 4.1) and decision rules (Sec. 4.2). Although most part of the summarized algorithms presented in this paper were not developed aiming at performing feature drift detection and adaptation, we discuss them and highlight their capabilities to attack this problem, either via randomness (Sec. 4.3), combinatorics (Sec. 4.4) or windowing (Sec. 4.5).

\subsection{Decision Tree Learning}

Learning with decision trees is a predictive approach used in statistics, data mining and machine learning. In its simplest implementations, each internal node contains a test on a feature $D_{i} \in \mathcal{D}$, each branch from a node corresponds to an outcome of the test and each leaf contains a possible prediction (class value from $Y$ ) [14].

Predictions for instances $\vec{x}$ are obtained by traversing the tree with features' values, determining which branch should be followed, until a leave is reached.

Decision trees are learned by recursion, replacing leaves by test nodes, starting at the root. The feature of each test node is chosen by comparing all the available attributes $D_{i} \in \mathcal{D}$ according to some heuristic measure. 
Table 1: Summary of existing algorithms that perform feature selection during stream learning.

\begin{tabular}{|c|c|c|c|c|c|}
\hline Algorithm & $\begin{array}{l}\text { Learning } \\
\text { Approach }\end{array}$ & $\begin{array}{l}\text { Feature Selection } \\
\text { Algorithm }\end{array}$ & $\begin{array}{c}\text { Feature Drift } \\
\text { Adaptation Method }\end{array}$ & $\begin{array}{l}\text { Explicit Dynamic } \\
\text { Feature Selection }\end{array}$ & Reference \\
\hline VFDT & Tree & $\begin{array}{c}\text { Entropy } \\
\text { Information Gain } \\
\text { Gini Coefficient }\end{array}$ & - & & {$[37]$} \\
\hline Facil & Rules & Purity & - & & {$[42]$} \\
\hline VFDR & Rules & Entropy & - & & {$[3]$} \\
\hline Streaming Random Forest & Ensemble (Trees) & - & Randomness/Combinatorics & & {$[43,19]$} \\
\hline Random Rules & Ensemble (Rules) & - & Randomness/Combinatorics & & {$[15]$} \\
\hline Streaming Stacking & - & Ensemble & Combinatorics & & [44] \\
\hline CVFDT & Tree & $\begin{array}{c}\text { Entropy } \\
\text { Information Gain } \\
\text { Gini Coefficient }\end{array}$ & Windowing & $\checkmark$ & {$[45]$} \\
\hline HEFT-Stream & Ensemble & FCBF & Windowing & $\checkmark$ & {$[21]$} \\
\hline HAT & Tree & $\begin{array}{c}\text { Entropy } \\
\text { Information Gain } \\
\text { Gini Coefficient }\end{array}$ & Windowing & $\checkmark$ & {$[46]$} \\
\hline HUWRS & Ensemble & - & Windowing & & {$[47]$} \\
\hline
\end{tabular}

\subsubsection{Very Fast Decision Tree}

The Very Fast Decision Tree (VFDT) algorithm constructs decision trees by using constant memory and constant time per sample [37]. Trees are built by recursively replacing leaves with decision nodes, as data arrives. Different heuristic evaluation functions are used to determine whether a split should be performed or not, such as Entropy (Eq. 7), Correlation (Eq. 8), Information Gain (Eq. 9) and Gini Impurity (Eq. 10) [48], where $n$ is the amount of instances in the dataset analyzed.

$$
\begin{gathered}
H\left(D_{i}\right)=-\sum_{q \in D_{i}} P[q] \log _{2} P[q] \\
C\left(D_{i}, Y\right)=\frac{\sum_{q \in D_{i}} \sum_{y_{i} \in Y}\left(q-\bar{D}_{i}\right)\left(y_{i}-\bar{Y}\right)}{\sqrt{\sum_{q \in D_{i}}\left(q-\bar{D}_{i}\right)^{2}} \sqrt{\sum_{y_{i} \in Y}\left(y_{i}-\bar{Y}\right)^{2}}} \\
I G\left(D_{i}\right)=H\left(D_{i}\right)-\prod_{D_{j} \in \mathcal{D}, D_{j} \neq D_{i}} \frac{H\left(D_{j}\right)}{n} \\
G I\left(D_{i}\right)=1-\sum_{q \in D_{i}}(P[q])^{2}
\end{gathered}
$$


In order to determine whether a new branch should be built in the tree, VFDT assumes that the input data meets the Hoeffding bound [49].

Definition 4.1. The Hoeffding Inequality states that with probability $(1-\delta)$ the true mean of a variable is at least $(\bar{r}-\epsilon)$, where $\epsilon$ is given by Eq.11, $\delta$ is a user-given confidence bound, $r \in \mathbb{R}^{+}$is a random variable with range $R, n$ is the number of independent observations and $\bar{r}$ is the mean computed by the latter observations.

$$
\epsilon=\sqrt{\frac{R^{2} \ln \left(\frac{1}{\delta}\right)}{2 n}}
$$

The Hoeffding bound is able to give results regardless of the probability distribution that generates data. However, the number of observations needed to reach certain values of $\delta$ and $\epsilon$ are different across different probability distributions [50], therefore, it must be seen as a pessimistic bound. Generally, with

probability $(1-\delta)$, one can say that one attribute is superior when compared to others when the observed difference of information gain (or any other heuristic metric that computes the importance of an attribute) is greater then $\epsilon$.

Although VFDT performs embedded feature selection in data streams, it assumes that the distribution generating data does not change over time, therefore, it does not detect nor adapt to possible drifts.

\subsection{Decision Rule Learning}

Although decision trees account for readability, in some specific scenarios, where trees tend to grow large, they become hard to understand since nodes appear in a specific context defined by tests at antecedent nodes [15]. In contrast, classifiers based on rules have the advantage of both modularity and interpretability, where each rule is independent of the others and can be interpreted in isolation from any other rules.

A decision rule is a logic predicate in the $\boldsymbol{I F}$ antecedent $\boldsymbol{T H E N}$ label form, where the antecedent is a conjunction of conditions over features $D_{i} \in \mathcal{D}$ and the label is a possible class value that belongs to $Y$. 


\subsubsection{Facil}

The first streaming rule learner published was Facil [42]. Facil creates rules according to the arrival of instances in an incremental fashion. In order to cope with concept drifts, Facil encompasses both explicit and implicit forgetting mechanisms. The explicit approach occurs when the examples are older than a user-given threshold $W$, adopting a sliding window approach to eliminate old rules. Conversely, implicit forgetting occurs when removing rules that are not relevant as they do not enforce any concept description boundary. This approach's rationale is that rules are inconsistent if they store both positive and negative instances that are near to one another at the decision boundary. Therefore, rules are removed if the impurity (ratio between positive instances it covers and its total number of cover examples) of a rule reaches a user-given threshold. Whenever the removal of a rule occurs, the subset originally covered by these rules are used to form two new rules that achieve satisfiable purity.

\subsubsection{Very Fast Decision Rules}

A more robust approach for learning rules from data streams is proposed in [3]. This algorithm, called Very Fast Decision Rules (VFDR), is capable of learning ordered and/or unordered rules. The algorithm starts with an empty rule set and rules are grown and expanded according to the minimization of entropy (Eq. 7) of class labels $Y$ of instances covered by each rule. Additionally, in order to determine whether a rule should be expanded, VDFR also adopts the Hoeffding bound (Eq. 11).

VFDR considers two cases of rule learning: ordered and unordered sets of rules. In the former, all labeled instances update statistics of the first rule triggered. While in the latter, labeled instances update statistics of all the rules that cover it. In both cases, if no rules cover an instance, the default rule is updated to cover them.

Finally, VFDR encompasses two classification strategies. The first uses only information about class distribution and does not account for features' values. Since it uses a small part of the available information, it is a crude approx- 
imation of the instances. Conversely, in an informed strategy, instances are classified with the class that maximizes the posteriori probability assuming the independence of attributes given the class $\left(P\left[y_{i} \mid \vec{x}\right] \propto P\left[y_{i}\right] \prod P\left[\vec{x} \mid y_{i}\right]\right)$.

\subsection{Randomness}

Diversity is a trait of a variety of recently proposed algorithms for learning from data streams $[51,52,53]$, particularly ensembles. Ensembles are sets of classifiers that are trained in parallel or in sequence and have their predictions aggregated during polling [54]. In several of these approaches, experts of an ensemble are trained with different inputs in order to promote diversity [55]. A well-known approach for inducing diversity in ensembles is Bagging [56]. Originally, a bagging ensemble is composed of $m$ classifiers, which are trained with subsets (bootstraps) of the whole training set. However, sampling usually is not feasible in a data stream configuration, since that would require storing all instances before creating subsets. Therefore, authors in [53] observed that the probability of an instance $\vec{x}_{i}$ to be selected for a subset can be approximated by a Poisson distribution with $\lambda=1$.

Although promoting diversity through instances is an interesting approach to enhance a learner's accuracy, more recent approaches aim at promoting diversity through different feature subsets, i.e. vertical partitioning of data $[43,19]$. By learning through ensembles with different features, experts learn partially (or completely) disjoint areas of the feature space, resulting in a highly diverse ensemble. Although these algorithms do not focus explicitly on adapting to feature drifts, they do present implicit adaptation to this characteristic of data streams by covering different feature subspaces in parallel.

\subsubsection{Streaming Random Forest}

The Streaming Random Forest classifier is an adaptation of the ensemblebased Random Forest classifier [57]. Random forests are ensembles of decision trees. Assuming a dataset with $n$ instances, each belonging to a feature set $\mathcal{D}$, random forests grow a set of trees, each using a bootstrap sample drawn from 
the full training set. Bootstrapping guarantees that about $\frac{n}{3}$ of the records are not included in the training set and so are available for evaluation of each tree [19].

The construction of each tree follows a variant of the typical decision tree building algorithm. In standard decision tree algorithms, the set of attributes considered at a node is the entire set $\mathcal{D}$. Conversely, in the Random Forest algorithm, the set of attributes considered at each node of each tree of the ensemble is a different randomly chosen subset $\mathcal{D}^{\prime} \subset \mathcal{D}$, where $\left|\mathcal{D}^{\prime}\right| \leq M$.

As an ensemble, the labeling of each new instance is the fusion of the votes of all the trees. The random forest classification error depends on (i) the correlation among its component trees, since smaller correlations cause higher variance canceling in voting and (ii) the strength of each individual tree, since the more accurate each subtree is, the better its individual vote and the smaller the error rate is [43].

Therefore, the value of $M$ is a sensitive parameter of random forests that must be chosen carefully. Small values of $M$ tend to increase the strength of each individual tree, while decreasing the correlation between them [43].

\subsubsection{Random Rules}

In [15], authors extend the VFDR algorithm by promoting randomness. This algorithm, called Random Rules for Data Stream (RR), encompasses the following parameters: a number of rule sets $\left(N_{s}\right)$ and the number of attributes $M$ that respects the $M<|\mathcal{D}|$ restriction.

Initially, each of the composing rule sets is empty and each of these is associated with a random subset $\mathcal{D}^{\prime} \subset \mathcal{D}$ of size $M$. For each instance $i_{t}$ retrieved from $\mathcal{S}$, RR generates a random number $p$ between 0 and 1 for each rule set. If $p \geq T_{r n d}$, a user-given threshold, $\mathrm{RR}$ verifies whether each rule set contains a rule that covers $i_{t}$, i.e. if all the literals of the rule are true for the given instance. If the above condition holds, all covering rules are expanded using only the features adopted by the rule set $\mathcal{D}^{\prime}$. Otherwise, that is, if no rules cover $i_{t}$, then the default rule is updated to cover it, again, respecting the features in $\mathcal{D}^{\prime}$. 
Finally, authors presented two voting schemes. The first classifies $\vec{x}_{t}$ with the class $y_{i}$ that maximizes $P\left[y_{i}\right]$, while the second assumes the class that maximizes the posteriori probability $\max _{y_{i} \in Y} P\left[y_{i} \mid \vec{x}_{t}\right]$ presented in Eq. 1 .

\subsection{Combinatorics}

By exploring combinatorics, ensembles of decision trees and random rules algorithms can be extended and posed as dynamic wrappers for dynamic feature selection for data streams. If one assumes an ensemble of decision trees or a random rule algorithm, where each of its containing experts is trained with a different subset of the entire feature set $\mathcal{D}$, and that the cardinality of each subset is at maximum $M$, the ensemble would contain $\sum_{i=1}^{M}\left(\begin{array}{c}M \\ i\end{array}\right)$ experts. Although training this high amount of experts is computationally expensive in terms of both processing time and memory space, it guarantees that a near optimal (or optimal, if $\left.M \geq\left|\mathcal{D}^{*}\right|\right)$ subset $\mathcal{D}^{\prime}$ allocated to one of the experts will maximize its acuity metric [19]. Therefore, by applying weighted majority voting [58], feature drifts can be detected according to the increase of the weights of experts with the current most discriminative subsets of features, while those with subsets of irrelevant features will possess lower weights due to lower accuracy performance.

\subsubsection{Streaming Stacking}

In [44], authors produce a classification model based on an ensemble of decision trees, each of which is built from a random and distinct subset of $\mathcal{D}^{\prime} \subset \mathcal{D}$. The overall model is formed by combining the log-odds of the class probabilities of its containing trees using sigmoid perceptrons, with one perceptron per class. Contrarily to the conventional boosting approach, which forms an ensemble in a greedy fashion, each tree is built in sequence by assigning weights as a byproduct and their method generates trees in parallel and combines them using perceptron classifiers by applying stacking [59]. Due to the streaming scenario, VFDTs are used as ensemble members since they are able to be trained incrementally. Additionally, the ensemble adopts the ADWIN change detector [50] in order to detect and adapt to possible concept drifts. 


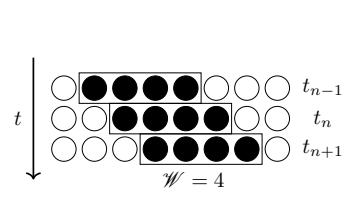

(a) Sliding.

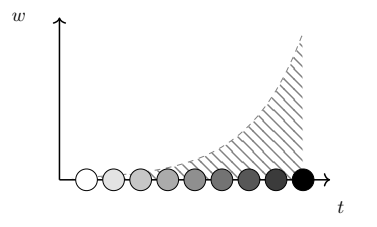

(b) Damped.

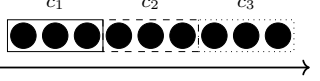

(c) Landmark.

Figure 3: Window models. Adapted from [20].

This approach is based on generating trees for all possible feature subsets of a given size $M$. Assuming a feature set $\mathcal{D}$ of size $d$, there are $\left(\begin{array}{c}d \\ M\end{array}\right)$ possible subsets. Clearly, only moderate values of $M$ or values close to $d$ are practical, since $\left(\begin{array}{c}d \\ M\end{array}\right)=\left(\begin{array}{c}d \\ d-M\end{array}\right)$. Authors claim that $M=2$ is very practical for datasets with a moderate number of features, although certainly not feasible for highdimensional data (e.g. Spam Corpus [39]).

\subsection{Windowing}

A common approach for both data management and dealing with drifting data is to maintain a predictive model consistent with a set of recent examples [20] given three window models: sliding, damped and landmark. In all cases, the difficulty is to select their appropriate size due to the stability-plasticity dilemma. While short windows reflect the current data distribution and ensure fast adaptation to drifts (plasticity), shorter ones worsen the performance of the system in stable areas. Conversely, larger windows give better performance in stable periods (stability), however, they also respond more slowly to drifts [18].

Sliding windows (Fig. 3a) store in memory a fixed or variable amount $\mathscr{W}$ of recent examples. Whenever a new instance arrives, it is enqueued in a FIFO (first in, first out) policy data structure, where the oldest one is discarded. The rationale behind this type of window is that buffered data reflect the current concept adequately. In opposition to sliding windows, damped windows (Fig. b) associate a weight $w$ to each datum, which decays with time. This windowing technique is interesting because weights can be seen as indicatives of how important an instance is to the current concept, thus, may be accounted 
for during voting. Finally, landmark windows require processing a stream by handling disjoint chunks $c_{i}$ of data separately by instances called "landmarks". Landmarks can be defined in terms of time, in terms of the number of instances seen since the previous landmark or accordingly to memory constraints. All instances belonging to a same landmark window are stored or summarized into a same data structure, which is used for training. In this section we present existing works that rely in windowing approaches to explicitly adapt to feature drifts.

\subsubsection{Concept-adapting Very Fast Decision Tree}

Concept-adapting Very Fast Decision Tree (CVFDT) is an extension to VFDT to deal with concept drifts [45]. CFVDT keeps a model consistent with respect to the current state of a sliding window from the data stream, thus creating and replacing alternate decision subtrees when it detects that the distribution of data is changing at a node. As instances $i_{t}$ arrive, CFVDT updates the statistics at its nodes by decrementing counters according to the oldest element in the window, which is about to be dequeued and "forgotten".

Therefore, CFVDT is an Hoeffding Tree which periodically verifies the statistics of nodes to determine if the Hoeffding criterion is still met. According to user-given parameters $T_{0}, T_{1}$ and $T_{2}$, CFVDT traverses the entire decision tree and checks at each test node if the splitting attribute is still the best when compared to others. If there is an alternate better splitting attribute, the entire subtree is replaced by a new split node with this attribute. Later, during the next $T_{1}$ instances, all retrieved instances from $\mathcal{S}$ are used to build the new subtree, which are then tested with the following $T_{2}$ instances.

\subsubsection{Heterogeneous Ensemble for Data Stream}

The Heterogeneous Ensemble with Feature Drift for Data Stream (HEFTStream) is an algorithm that incorporates feature selection into an heterogeneous ensemble to adapt to different types of concept and feature drifts [21]. HEFT-Stream adopts an modification of the Fast Correlation-Based Filter 
(FCBF) algorithm so it dynamically updates the selected relevant feature subset of a data stream.

FCBF is a feature selection algorithm where the class relevance and pairwise dependencies between features are accounted for. Based on information theory, FCBF adopts symmetrical uncertainty $(S U)$ to compute dependencies of features and class relevance. Using a top-down approach, starting from the whole feature set $\mathcal{D}$, FCBF heuristically applies a backward selection technique to remove irrelevant and redundant features.

Symmetrical uncertainty uses both entropy and conditional entropy to calculate the dependencies of features. Assuming two arbitrary features $D_{i}$ and $D_{j}$, the symmetrical uncertainty between these two can be computed according to Eq. 12, where $H(\cdot)$ is the entropy of a feature (Eq. 7), $H(\cdot, \cdot)$ is the conditional entropy and $M I(\cdot, \cdot)$ is the mutual information between two features (Eq. 13).

$$
\begin{gathered}
S U\left(D_{i}, D_{j}\right)=2\left[\frac{H\left(D_{i}\right)-H\left(D_{i} \mid D_{j}\right)}{H\left(D_{i}\right)+H\left(D_{j}\right)}\right]=2\left[\frac{M I\left(D_{i}, D_{j}\right)}{H\left(D_{i}\right)+H\left(D_{j}\right)}\right] \\
M I\left(D_{i}, D_{j}\right)=\sum_{q \in D_{i}} \sum_{r \in D_{j}} P[q, r] \log \frac{P[q, r]}{P[q] \times P[r]}
\end{gathered}
$$

HEFT-Stream adopts a landmark windowing approach. Incoming data is stored in a buffer with a predefined size. Next, the matrix of symmetrical uncertainty values is computed to select the most relevant feature subset. After the processing of each data chunk, HEFT-Stream postulates that a feature drift has occurred if two consecutive selected subsets of features differ.

Additionally, in order to boost the overall ensemble accuracy, HEFT-Stream promotes diversity among member classifiers by encompassing an Online Bagging sampling procedure [53].

Classification of each instance is performed through a weighted combination of member classifiers classifications. Each member classifier $k$ is associated to a weight $w_{k}$ (Eq. 14) which is an accumulated error from its creation time to the current time. The weight $w_{k}$ is stated in Eq. 14 where $\alpha$ is a padding value 
which was originally empirically set to 0.001 and $E_{k}$ is the accumulative error of the $k^{\text {th }}$ member classifier.

$$
w_{k}=\frac{1}{\left(E_{k}+\alpha\right)} \times\left[\sum_{m=1}^{K}\left(E_{m}+\alpha\right)^{-1}\right]
$$

Finally, at the end of a chunk, the classifier with the highest value of $E_{k}$ is replaced by a new classifier. This new classifier is associated with the feature set $\mathcal{D}^{\prime}$ selected by FCBF and its type corresponds to the most accurate expert of the ensemble.

Although HEFT-Stream is stated as a generic ensemble capable of using any kind of base classification learners, authors only show results for a combination of an Updatable Naïve Bayes algorithm and VFDT.

\subsubsection{Hoeffding Adaptive Tree}

Most of decision tree-based algorithms for learning from data streams either assume that the underlying distribution is static, e.g. VFDT (see Sec. 4.1.1), or contain hardwired constants concerning the speed or frequency of change, e.g. CVFDT (see Sec. 4.5.1). These choices are inconclusive and often incorrect due to the plasticity-stability dilemma, but also since one cannot assume that all changes in a stream occur with the same frequencies and lengths.

In [46] authors proposed the adoption of an adaptive sliding window drift detector, named ADWIN [50], inside decision trees for data streams. Their proposal, called Hoeffding Adaptive Tree (HAT), is an extension to CVFDT in which an ADWIN drift detector is used to monitor and flag changes in split nodes of the tree. Therefore, instead of relying on window parameters $T_{0}, T_{1}$ and $T_{2}$ for re-evaluating split nodes, HAT replaces split nodes when a significant error rate change occurs, given a confidence level $\delta$ that is inputted to ADWIN.

HATs are thus able to cope with both concept drifts and feature drifts since split nodes are re-evaluated. This allows split nodes to be consistent in terms of the feature adopted to perform the split and in which range/value of this feature the decision should be made. One of the major drawbacks of this method is 
that ADWIN is known for triggering too many false positives [60], i.e. it flags changes when they do not really occur.

\subsubsection{Heuristic Updatable Weighted Random Subspaces}

The Heuristic Updatable Weighted Random Subspaces (HUWRS) is a random subspace-based ensemble for data streams [47]. HUWRS works under the hypothesis that when a feature drift occurs, there is no need to learn an entirely new predictive model. Instead, authors recommend building experts of the ensemble based on random subspaces, while feature drifts are detected accordingly to a landmark window. HUWRS assumes that data arrives in batches. On each arriving batch, features are discretized in equal-sized bins and the class distribution inside each bin of every feature is computed.

HUWRS postulates that a feature drift occurs in a feature $D_{i}$ if the Hellinger weight between the class distribution of the current and prior landmarks differ at least by $p \%$, a user-given threshold. The Hellinger weight is given by Equation 15, which is a normalization to the Hellinger distance, given by Equation 16. In Equations 15 and $16, Y^{\prime}$ and $Y^{\prime \prime}$ stand for the class distributions of the current and prior landmarks for an arbitrary feature $D_{i}$.

$$
\begin{gathered}
w_{H}\left(Y^{\prime}, Y^{\prime \prime}\right)=\frac{\sqrt{2}-d_{H}\left(Y^{\prime}, Y^{\prime \prime}\right)}{\sqrt{2}} \\
d_{H}\left(Y^{\prime}, Y^{\prime \prime}\right)=\sqrt{\sum_{q \in D_{i}}\left(\sqrt{P\left[Y^{\prime} \mid D_{i}=q\right]}-\sqrt{P\left[Y^{\prime \prime} \mid D_{i}=q\right]}\right)^{2}}
\end{gathered}
$$

Since a low Hellinger distance means a high agreement in the two distributions, a low Hellinger distance should correspond to a high weight. We emphasize that $\sqrt{2}$ is the maximum Hellinger distance between distributions for binary classification problems, thus, this value is used as a normalization factor so that the Hellinger weight is bounded in $[0 ; 1]$.

Whenever a feature drift is flagged for a feature $D_{i}$, HUWRS resets only the experts associated with such feature. Therefore, HUWRS is expected to adapt to feature drifts while performing less retraining when compared to full reset 
approaches. One important drawback of HUWRS is that the experts adopted in the ensemble are not incremental. Therefore, classifiers are unable to increment their models if conventional concept drifts occur.

\section{Empirical Analysis}

In this section we assess the impact of feature drifts on data stream classification algorithms. First, we introduce the experimental protocol adopted, including data generators, one real-world dataset and statistical procedures (Sec. 5.1). Later, we discuss the results obtained, highlighting existing algorithms difficulties to overcome feature drifts (Sec. 5.2). Finally, we propose a naive solution to the feature drift problem by splitting and treating the stream into disjoint chunks of data and applying simple filters. We also show the efficiency of this approach in both synthetic and real world problems, thus, highlighting the need for future research in this area and the room for more sophisticated approaches (Sec. 5.3).

\subsection{Experimental Protocol}

In this section we present the experimental protocol adopted. We start by presenting the datasets used and later the evaluation procedure, focusing on accuracy, processing time and memory usage metrics and statistical testing procedure.

\subsubsection{Generators}

In order to evaluate whether a learning algorithm is able to work in different scenarios, it is necessary to assess its performance over different datasets. In opposition to real-world data, synthetic data stream generators are important and often used due to their flexibility, since they offer a precise definition of drifts types and locations during the streams. In this section we propose and survey generators capable of synthesizing feature drifts, thus, enabling proper

evaluation of learning algorithms in these scenarios. All the values picked for 
presented parameters were chosen accordingly to their usage in previous papers of the area.

$S E A-F D$. In [38], authors proposed a data stream generator that extends the SEA generator [61]. SEA-FD simulates streams with $d>2$ uniformly distributed features given by the user, where $\forall D_{i} \in \mathcal{D}, D_{i} \in[0 ; 10]$ and only two randomly picked features are relevant to the concept to be learned: $\mathcal{D}^{*}=$ $\left\{D_{\omega}, D_{\zeta}\right\}$. As in $[61,38]$, the class value $y$ is defined by Eq. 17, where $\theta$ is a user-given threshold. In our experiments, $\theta=7$ and each instance synthesized has a $5 \%$ probability of being generated as noise.

$$
y= \begin{cases}1, & \text { if } D_{\alpha}+D_{\beta} \leq \theta \\ 0, & \text { otherwise }\end{cases}
$$

$B G-F D$. The Binary Generator with Feature Drift (BG-FD) generates instances composed by boolean $(\{0,1\})$ features. BG-FD has three functions: BG1-FD, BG2-FD and BG3-FD, all inspired by [31]. In BG1-FD, from the entire set of features $\mathcal{D}$, only a random subset $\mathcal{D}^{*} \subset \mathcal{D}$ is relevant to the concept to be learned. Additionally, $\left|\mathcal{D}^{*}\right|=d_{r}$, where $d_{r}$ is a user-given parameter. Conversely, in BG2-FD and BG3-FD we have $\mathcal{D}^{*}=\left\{D_{\alpha}, D_{\beta}, D_{\epsilon}\right\}$. Labels of instances are given according to three different functions presented in Eqs. 18, 19 and 20 for BG1-FD, BG2-FD and BG3-FD, respectively.

$$
\begin{gathered}
y= \begin{cases}1, & \text { if } \bigwedge_{D_{i} \in \mathcal{D}^{*}} D_{i} \\
0, & \text { otherwise }\end{cases} \\
y= \begin{cases}1, & \text { if }\left(D_{\alpha} \wedge D_{\beta}\right) \vee\left(D_{\alpha} \wedge D_{\epsilon}\right) \vee\left(D_{\beta} \wedge D_{\epsilon}\right) \\
0, & \text { otherwise }\end{cases} \\
y= \begin{cases}1, & \text { if }\left(D_{\alpha} \wedge D_{\beta} \wedge D_{\epsilon}\right) \vee\left(\neg D_{\alpha} \wedge \neg D_{\beta} \wedge \neg D_{\epsilon}\right) \\
0, & \text { otherwise }\end{cases}
\end{gathered}
$$


In all cases, class labels $y_{i} \in Y$ are evenly likely to occur and instances have a $5 \%$ probability of being generated as noise.

$R T G-F D$. The original Random Tree Generator (RTG) builds a decision tree by randomly performing splits on features and assigning a random class label to each leaf [62]. Instances are created by generating a random valued $\vec{x}$ and traversing the tree for its corresponding label. We propose an extension to this generator, namely RTG-FD, such that only a random subset of features $\mathcal{D}^{*} \subset \mathcal{D}$ are relevant. Assuming $\mathcal{D}_{i}=\mathcal{D} \backslash \mathcal{D}^{*}$ as the subset of irrelevant features, $\left|\mathcal{D}_{i}\right|$ is a user-given parameter.

\subsubsection{Drift Framework}

We synthesize feature drifts in streams accordingly to the framework proposed in [14]. This framework models a drift as the change between two pure distributions, each given by a distinct concept. Intuitively, at the beginning of a drift window there is a higher probability that instances belong to the prior concept $C_{A}$. As we move towards its end, the probability that an instance belongs to the posterior concept $C_{B}$ increases. The drift window ends when concept $C_{B}$ becomes stable. To model the probability that every new instance $i_{t}$ drawn from $\mathcal{S}$ belongs to concept $C_{A}$ or $C_{B}$, a sigmoid function as stated in Eq. 21 is adopted, where $P\left[C_{B}\right]$ and $P\left[C_{A}\right]=\left|1-P\left[C_{B}\right]\right|$ are, respectively, the probabilities of $i_{t}$ belonging to $C_{A}$ or $C_{B}, W$ is the drift window size, $t$ is the current timestamp and $t_{0}$ is the time of the drift, i.e. when $P\left[C_{A}\right]=P\left[C_{B}\right]$ holds.

$$
P\left[C_{B}\right]=\left|1-P\left[C_{A}\right]\right|=\left(1+e^{-W\left(t-t_{0}\right)}\right)^{-1}
$$

Therefore, with the latter generators, feature drifts occur when the relevant subset of features $\mathcal{D}^{*}$ of $C_{A}$ differs from the relevant subset of features $\mathcal{D}^{*}$ of the subsequent concept $C_{B}$.

Synthetic data streams. Synthetic experiments encompass the usage of all presented generators. All streams created have a length of 100,000 instances, 
$|\mathcal{D}|=50$ and $\left|\mathcal{D}^{*}\right|=3$, with the exception of SEA-FD experiments, where $\left|\mathcal{D}^{*}\right|=2$. Streams with an (A) suffix contain 9 equally distributed abrupt $(w=1)$ feature drifts, while streams with a $(\mathrm{G})$ contain 9 drifts at the same time points as for $(\mathrm{A})$, however, these drifts are gradual $(w=1,000)$.

Real datasets. Complementing the synthetic data streams, our experiments also encompass the Spam Corpus dataset [39]. This dataset (earlier discussed in Sec. 3.4) was extracted from a text mining process on an online news dissemination system. The Spam Corpus dataset contains 9,324 instances and 39,917 features, such that each attribute represents the presence of a single word (feature) in the instance (e-mail). Also, this dataset is known for containing a concept drift which occurs gradually around the instance number 1,500 [39, 10].

\subsubsection{Evaluation Procedure}

Our evaluation procedure assesses an algorithm's efficiency in terms of accuracy, processing time and memory usage. To quantify the accuracy of classifiers, we adopted the Prequential test-then-train procedure [63]. Although the Prequential evaluation is known for being pessimistic, authors in [63] claim that it converges to a periodic holdout estimate when estimated over a sliding window. The Prequential accuracy of a classifier is computed, at a timestamp $t_{i}$, over a sliding window of size $w^{\prime}$, according to Eq. 22, where $L(\cdot, \cdot)$ is a loss function (in our case, we adopted a 0-1 function) for the obtained class value $y_{k}$ and the expected $\hat{y}_{k}$.

$$
P_{w^{\prime}}\left(t_{i}\right)=1-\frac{1}{w^{\prime}} \sum_{k=i-w^{\prime}+1}^{i} L\left(y_{k}, \hat{y}_{k}\right)
$$

Processing time is measured as the time that the algorithms spends processing in seconds, and memory usage is presented in RAM-Hours, where 1 RAM-Hour equals 1 GB of RAM being used for one hour.

All experimental results presented in this paper were obtained on a Intel Xeon CPU E5649 @ 2.53GHz $\times 8$ based computer running CentOS with 16GB of memory and under the Massive Online Analysis (MOA) framework [62]. 
Finally, in order to determine whether there is significant statistical difference between algorithms, Wilcoxon's test [64] or a combination of Friedman's [65] and Nemenyi's [66] non-parametric hypothesis tests are used, according to the number of evaluated hypotheses.

\subsection{Benchmarking Existing Works}

In this section we present the results for the following algorithms: Very Fast Decision Rules (VFDR), Very Fast Decision Tree (VFDT), Hoeffding Adaptive Tree (HAT), Random Rules (RR), Streaming Random Forest (SRF), HEFTStream (HEFT) and Streaming Stacking (SS) (all surveyed in Sec. 4), an 1Nearest Neighbor algorithm (1NN) and an Updatable Naïve Bayes (NB).

Tab. 2 presents the average prequential accuracy results obtained during experiments for all the algorithms. We highlight the higher results obtained by HAT and ensemble-based approaches, which outperformed its base learners in most cases. This highlights the power of HAT and ensemble-based algorithms to perform feature drift detection, either via combinatorics, randomness or windowing.

In Fig. 4 we present the accuracy of the best and worst ranked algorithms during the SEA-FD(A), SEA-FD $(G)$ and Spam Corpus experiments, where one can see that HAT is able to quickly recover from feature drifts and boosts accuracy even after them. Specifically in Fig. 4c, one can see that HAT is capable of detecting the feature drift, therefore quickly adapting to it while the Updatable Naïve Bayes (NB) slowly recovers from it only after half the experiment.

Tabs. 3 and 4 present results obtained for processing time and RAM-Hours, respectively, where one can see that ensemble-based algorithms possess higher processing time and memory usage, as expected. We emphasize that both Random Rules and Streaming Random Forest with $M=2$ were incapable of performing in the Spam Corpus dataset, due to insufficient memory space $(>16 G B)$. 
Table 2: Average accuracy obtained during experiments.

\begin{tabular}{cccccccccccc}
\hline \multicolumn{10}{c}{ Average Accuracy $(\%)$} \\
\hline Experiment & NB & VFDR & VFDT & HAT & $1 N N$ & $\begin{array}{c}\text { RR } \\
(M=1)\end{array}$ & $\begin{array}{c}\text { RR } \\
(M=2)\end{array}$ & $\begin{array}{c}\text { SRF } \\
(M=1)\end{array}$ & $\begin{array}{c}\text { SRF } \\
(M=2)\end{array}$ & HEFT & SS \\
\hline RTG-FD(A) & 55.43 & 56.43 & 55.65 & 64.19 & 57.21 & 65.98 & $\mathbf{6 6 . 3 5}$ & 65.56 & 65.42 & 59.62 & 56.73 \\
RTG-FD(G) & 55.41 & 55.86 & 55.68 & 63.33 & 57.14 & 65.78 & $\mathbf{6 6 . 3 7}$ & 65.56 & 65.21 & 62.13 & 56.13 \\
SEA-FD(A) & 79.83 & 78.92 & 80.82 & 83.73 & 75.28 & 95.57 & 95.65 & 98.29 & $\mathbf{9 8 . 5 9}$ & 83.02 & 75.37 \\
SEA-FD(G) & 78.92 & 77.62 & 80.80 & 84.16 & 75.28 & 93.23 & 93.32 & 96.97 & $\mathbf{9 7 . 3 9}$ & 83.22 & 75.21 \\
BG1-FD(A) & 69.99 & 72.67 & 78.21 & $\mathbf{9 4 . 0 4}$ & 76.00 & 78.50 & 76.37 & 78.17 & 78.15 & 90.07 & 92.16 \\
BG1-FD(G) & 69.99 & 70.21 & 78.25 & $\mathbf{9 3 . 2 0}$ & 75.69 & 78.30 & 76.49 & 78.18 & 78.18 & 89.59 & 92.03 \\
BG2-FD(A) & 62.02 & 68.17 & 66.63 & $\mathbf{9 1 . 1 5}$ & 73.20 & 59.05 & 61.14 & 60.07 & 61.74 & 88.51 & 77.79 \\
BG2-FD(G) & 61.94 & 66.41 & 66.73 & $\mathbf{8 9 . 1 2}$ & 72.89 & 58.45 & 62.71 & 59.98 & 63.15 & 88.06 & 76.38 \\
BG3-FD(A) & 54.99 & 54.24 & 62.43 & 86.04 & 62.96 & 59.18 & 60.82 & 59.91 & 61.05 & $\mathbf{8 6 . 7 8}$ & 53.37 \\
BG3-FD(G) & 54.74 & 54.07 & 60.70 & 81.24 & 62.93 & 58.19 & 60.65 & 59.78 & 61.40 & $\mathbf{8 6 . 0 0}$ & 57.38 \\
Spam Corpus & 71.13 & 74.81 & 79.32 & $\mathbf{8 4 . 4 8}$ & 79.85 & 75.22 & - & 74.17 & - & 82.65 & - \\
\hline
\end{tabular}

In order to determine whether there is significant statistical difference between algorithms' accuracy, processing time and memory usage, we started with Friedman's test, while ignoring $\mathrm{RR}(M=2)$ and $\operatorname{SRF}(M=2)$ since they do not present accuracy values for all datasets. We divided our comparison in two distinct tests. The first test compares NB, VFDR, VFDT, HAT and $1 N N$, while the second compares ensemble-based algorithms, i.e. RR, SRF, HEFT and SS.

In our first test, Friedman test pointed out that there was a difference between algorithms by adopting a confidence level of $95 \%$ in terms of accuracy and the post-hoc Nemenyi test showed that $\{\mathrm{HAT}\} \succ\{1 N N$, VFDT, VFDR, NB\} also with a $95 \%$ confidence level. The same procedure was repeated for processing time and memory usage, and results show that $\{\mathrm{NB}$, VFDT, $1 N N\} \succ\{\operatorname{HEFT}, \operatorname{HAT}, \operatorname{SRF}(M=1)$, VFDR, RR $(M=1)\}$ for processing time and $\{\mathrm{NB}, 1 N N, \mathrm{VFDT}\} \succ\{\mathrm{VFDR}, \operatorname{SRF}(M=1), \mathrm{HAT}, \mathrm{HEFT}, \mathrm{RR}$ $(M=1)\}$ in terms of memory space.

In the second evaluation, Friedman's test pointed that $\{$ HEFT $\} \succ\{R R$ $(M=1), \operatorname{SRF}(M=1), \operatorname{SS}\}$ in terms of accuracy, however, there is no significant statistical between ensemble-based algorithms in processing time and memory usage. 


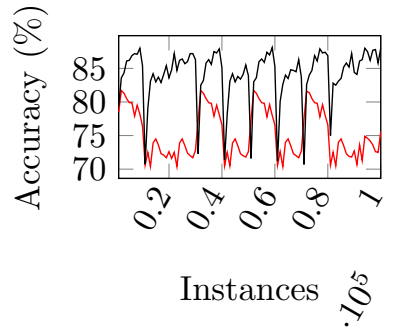

(a) SEA-FD(A)

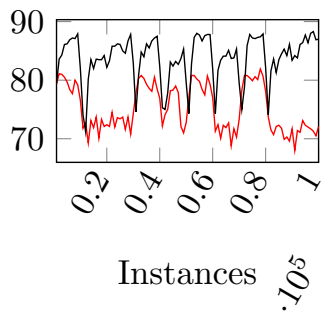

(b) SEA-FD(G)

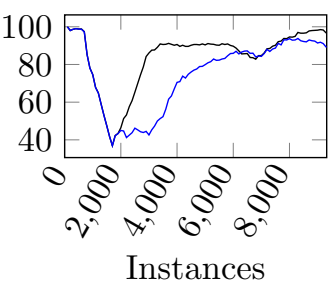

(c) Spam Corpus

\section{$1 N N-\mathrm{HAT}-\mathrm{NB}$}

Figure 4: Accuracy of the best and worst algorithms as the stream progresses.

Table 3: Processing time obtained during experiments.

\begin{tabular}{cccccccccccc}
\hline \multicolumn{10}{c}{ Processing time $(s)$} \\
\hline Experiment & NB & VFDR & VFDT & HAT & $1 N N$ & $\begin{array}{c}\text { RR } \\
(M=1)\end{array}$ & $\begin{array}{c}\text { RR } \\
(M=2)\end{array}$ & $\begin{array}{c}\text { SRF } \\
(M=1)\end{array}$ & $\begin{array}{c}\text { SRF } \\
(M=2)\end{array}$ & HEFT & SS \\
\hline RTG-FD(A) & $\mathbf{1 . 9 4}$ & 85.21 & 6.66 & 82.50 & 7.49 & 316.36 & 95.97 & 10.14 & 104.68 & 13.23 & 31.74 \\
RTG-FD(G) & $\mathbf{1 . 6 2}$ & 94.20 & 6.07 & 81.24 & 7.59 & 351.77 & 93.79 & 51.65 & 847.12 & 12.93 & 31.03 \\
SEA-FD(A) & $\mathbf{2 . 0 7}$ & 424.62 & 4.36 & 4.76 & 110.43 & 57.52 & 84.82 & 63.60 & 8598.08 & 29.01 & 30.61 \\
SEA-FD(G) & $\mathbf{2 . 0 7}$ & 192.48 & 4.68 & 3.61 & 110.38 & 58.72 & 79.43 & 65.10 & 8594.82 & 34.74 & 30.98 \\
BG1-FD(A) & $\mathbf{1 . 4 5}$ & 5.50 & 4.61 & 104.44 & 2.66 & 3700.78 & 112.59 & 45.47 & 576.54 & 5.20 & 27.75 \\
BG1-FD(G) & $\mathbf{1 . 4 3}$ & 5.54 & 4.44 & 103.24 & 2.86 & 3627.28 & 112.54 & 45.98 & 1036.78 & 5.47 & 27.82 \\
BG2-FD(A) & $\mathbf{1 . 5 9}$ & 7.55 & 4.04 & 104.88 & 3.18 & 2589.64 & 86.90 & 25.58 & 290.87 & 6.07 & 27.52 \\
BG2-FD(G) & $\mathbf{1 . 3 7}$ & 6.57 & 3.98 & 102.78 & 3.84 & 3756.22 & 89.38 & 49.93 & 1134.00 & 6.00 & 26.73 \\
BG3-FD(A) & $\mathbf{1 . 4 6}$ & 5.35 & 3.37 & 104.43 & 3.40 & 2330.19 & 86.94 & 25.52 & 295.29 & 5.89 & 27.88 \\
BG3-FD(G) & $\mathbf{1 . 3 3}$ & 5.75 & 3.67 & 102.54 & 4.09 & 3730.27 & 87.28 & 49.42 & 1145.98 & 5.74 & 26.81 \\
Spam Corpus & 617.66 & 691.51 & 695.06 & $\mathbf{1 4 5 . 2 3}$ & 6329.34 & 369.99 & - & 487.75 & - & 2132.02 & - \\
\hline
\end{tabular}

\subsection{Performing Feature Selection in Data Chunks}

In this section we propose and empirically evaluate a naive approach to handle feature drifts in data streams. We hypothesize that by splitting a stream into chunks, it is possible to determine the most discriminative subset of features of a stream, and train the classifier exclusively with them. In all cases, the most discriminative subset of features $\mathcal{D}^{*}$ is assumed as the union of features $D_{i} \in$ $\mathcal{D}$ that maximize the goodness function $Q\left(D_{i}\right)$ individually. We acknowledge that this discriminative subset selection is naive hence it does not account for redundant features [28]. This occurs due to all features being deemed relevant to 
Table 4: RAM-Hours obtained during experiments.

\begin{tabular}{|c|c|c|c|c|c|c|c|c|c|c|c|}
\hline \multicolumn{12}{|c|}{ RAM-Hours (GB-Hour) } \\
\hline Experiment & NB & VFDR & VFDT & HAT & $1 N N$ & $\begin{array}{c}\mathrm{RR} \\
(M=1)\end{array}$ & $\begin{array}{c}\mathrm{RR} \\
(M=2)\end{array}$ & $\begin{array}{c}\text { SRF } \\
(M=1)\end{array}$ & $\begin{array}{c}\text { SRF } \\
(M=2)\end{array}$ & HEFT & ss \\
\hline RTG-FD(A) & $1.76 \times 10^{-8}$ & $4.64 \times 10^{-4}$ & $1.85 \times 10^{-6}$ & $1.05 \times 10^{-5}$ & $1.18 \times 10^{-6}$ & $1.94 \times 10^{-4}$ & $2.90 \times 10^{-5}$ & $5.31 \times 10^{-7}$ & $8.22 \times 10^{-5}$ & $3.16 \times 10^{-6}$ & $1.82 \times 10^{-5}$ \\
\hline RTG-FD(G) & $1.62 \times 10^{-8}$ & $4.91 \times 10^{-4}$ & $1.81 \times 10^{-6}$ & $1.03 \times 10^{-5}$ & $1.21 \times 10^{-6}$ & $2.28 \times 10^{-4}$ & $3.58 \times 10^{-5}$ & $7.56 \times 10^{-6}$ & $2.29 \times 10^{-3}$ & $3.25 \times 10^{-6}$ & $1.82 \times 10^{-5}$ \\
\hline SEA-FD(A) & $1.56 \times 10^{-8}$ & $1.04 \times 10^{-2}$ & $6.08 \times 10^{-7}$ & $2.38 \times 10^{-7}$ & $1.36 \times 10^{-5}$ & $9.34 \times 10^{-5}$ & $3.05 \times 10^{-4}$ & $9.21 \times 10^{-5}$ & $1.10 \times 10^{-5}$ & $5.44 \times 10^{-2}$ & $2.11 \times 10^{-5}$ \\
\hline SEA-FD(G) & $1.42 \times 10^{-8}$ & $2.32 \times 10^{-3}$ & $6.20 \times 10^{-7}$ & $1.42 \times 10^{-7}$ & $1.36 \times 10^{-5}$ & $9.27 \times 10^{-5}$ & $3.68 \times 10^{-4}$ & $9.09 \times 10^{-5}$ & $7.87 \times 10^{-6}$ & $5.44 \times 10^{-2}$ & $2.16 \times 10^{-5}$ \\
\hline BG1-FD(A) & $1.21 \times 10^{-8}$ & $2.95 \times 10^{-7}$ & $7.36 \times 10^{-7}$ & $1.33 \times 10^{-5}$ & $3.99 \times 10^{-8}$ & $9.08 \times 10^{-3}$ & $3.48 \times 10^{-5}$ & $4.37 \times 10^{-6}$ & $3.86 \times 10^{-4}$ & $8.22 \times 10^{-7}$ & $5.57 \times 10^{-6}$ \\
\hline BG1-FD(G) & $1.29 \times 10^{-8}$ & $3.15 \times 10^{-7}$ & $7.25 \times 10^{-7}$ & $1.31 \times 10^{-5}$ & $4.71 \times 10^{-8}$ & $8.95 \times 10^{-3}$ & $3.50 \times 10^{-5}$ & $4.51 \times 10^{-6}$ & $9.79 \times 10^{-4}$ & $8.81 \times 10^{-7}$ & $5.60 \times 10^{-6}$ \\
\hline BG2-FD(A) & $1.32 \times 10^{-8}$ & $6.34 \times 10^{-7}$ & $4.91 \times 10^{-7}$ & $1.33 \times 10^{-5}$ & $6.70 \times 10^{-8}$ & $4.54 \times 10^{-3}$ & $2.10 \times 10^{-5}$ & $1.70 \times 10^{-6}$ & $1.30 \times 10^{-4}$ & $9.94 \times 10^{-7}$ & $5.59 \times 10^{-6}$ \\
\hline BG2-FD(G) & $1.23 \times 10^{-8}$ & $4.97 \times 10^{-7}$ & $4.45 \times 10^{-7}$ & $1.31 \times 10^{-5}$ & $8.04 \times 10^{-8}$ & $9.16 \times 10^{-3}$ & $2.61 \times 10^{-5}$ & $4.90 \times 10^{-6}$ & $1.07 \times 10^{-3}$ & $9.95 \times 10^{-7}$ & $5.35 \times 10^{-6}$ \\
\hline BG3-FD(A) & $1.32 \times 10^{-8}$ & $2.54 \times 10^{-7}$ & $2.89 \times 10^{-7}$ & $1.33 \times 10^{-5}$ & $7.57 \times 10^{-8}$ & $3.63 \times 10^{-3}$ & $2.13 \times 10^{-5}$ & $1.72 \times 10^{-6}$ & $1.32 \times 10^{-4}$ & $9.70 \times 10^{-7}$ & $5.68 \times 10^{-6}$ \\
\hline BG3-FD(G) & $1.20 \times 10^{-8}$ & $2.99 \times 10^{-7}$ & $3.65 \times 10^{-7}$ & $1.30 \times 10^{-5}$ & $1.19 \times 10^{-7}$ & $9.15 \times 10^{-3}$ & $2.53 \times 10^{-5}$ & $4.85 \times 10^{-6}$ & $1.08 \times 10^{-3}$ & $9.53 \times 10^{-7}$ & $5.33 \times 10^{-6}$ \\
\hline Spam Corpus & $2.34 \times 10^{-2}$ & $5.11 \times 10^{-2}$ & $1.56 \times 10^{-2}$ & $1.40 \times 10^{-3}$ & $5.50 \times 10^{-2}$ & $4.10 \times 10^{-3}$ & - & $4.46 \times 10^{-3}$ & - & $1.80 \times 10^{-1}$ & - \\
\hline
\end{tabular}

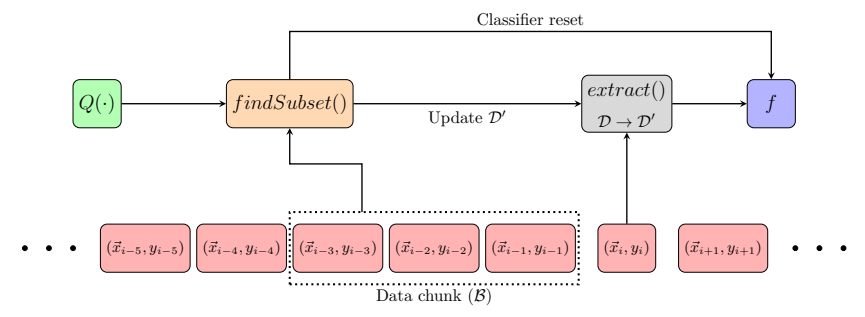

Figure 5: LFDD overview.

the concept (correlated with the class), while they are possibly highly correlated to one another [67]. Additionally, other studies suggest and empirically show that features with individual low discriminative power, when put together, are able to show interesting discriminative power at times [21, 35]. By performing feature selection as the stream progresses, we assume that a feature drift occurs when the most discriminative subset of features of a chunk of data differs from the subset of features of the previous chunk.

Our proposal is named Landmark-based Feature Drift Detector (LFDD). Its pseudocode is presented in Alg. 1 and an overview is depicted in Figure 5. It receives as input a data stream $\mathcal{S}$, a base learner $e$ (e.g. NB, VFDT, VFDR and $1 N N$ ), a landmark window size $W$, an heuristic goodness function $Q(\cdot)$ (e.g. Entropy, Gain Ratio and Information Gain) and a maximum amount of features $d_{\max }$.

During the training step, instances $\left(\vec{x}_{i}, y_{i}\right)$ retrieved from $\mathcal{S}$ are stored in an instance buffer $\mathcal{B}$ (lines 3 and 4 ) and used for training after the extraction of 
the most discriminative subset of features $\mathcal{D}^{\prime}$ (line 12).

When the size of the instance buffer reaches $W$ (line 5 ), then $\mathcal{D}^{\prime}$ is compared to the new most discriminant subset of features (line 6 ) given by a function findSubset $(\cdot, \cdot, \cdot)$, computed according to the instance buffer $\mathcal{B}$, to the heuristic goodness function $Q(\cdot)$ and the maximum amount of features $d_{\max }$ (line 6 ). If the new subset of discriminant of features $\mathcal{D}_{\text {new }}$ differs from the subset of the last chunk of data (line 7 ), we hypothesize that a feature drift has occurred, so the expert $e$ is reset and $\mathcal{D}^{\prime}$ is replaced with $\mathcal{D}_{\text {new }}$ (lines 8 and 9).

During the evaluation step, all instances are first translated into the reduced feature set $\mathcal{D}^{\prime}$ (line 13) and then the base learner is asked for a class label (line $14)$.

\subsubsection{Benchmarking LFDD}

In this section we evaluate the usage of LFDD in the Updatable Naive Bayes (NB), Very Fast Decision Tree (VFDT), Very Fast Decision Rules (VFDR) and a 1-Nearest Neighbor $(1 N N)$ algorithms. Our goal is to investigate if LFDD is able to improve overall accuracy of classification algorithms in feature drifting streams. We experimented with LFDD while varying the parameter $d_{\max }$ in the $[2 ; 49]$ interval and the following heuristic goodness functions: Correlation, Gain Ratio and Information Gain. We refrain from verifying the impact of the landmark window size $W$ since it represents a trade-off without a clear unique solution due to the plasticity-stability dilemma. A small size results in a window that reflects the current distribution of data and enables quicker drift adaptation (plasticity), while a large size enables a larger amount of data to work on, important in non-drifting periods of the stream (stability) [14]. Therefore, the size of the landmark window was empirically set to $W=1,000$ for synthetic experiments and $W=100$ for the Spam Corpus dataset.

In Figs. 6 and 7 we present the accuracy obtained by LFDD when varying the base learner, heuristic goodness function $Q(\cdot)$ and $d_{\max }$ in the $\operatorname{SEA-FD}(\mathrm{G})$ and Spam Corpus experiments, respectively. We do not provide the graphical results for other experiments since they follow the same behavior as those discussed in 


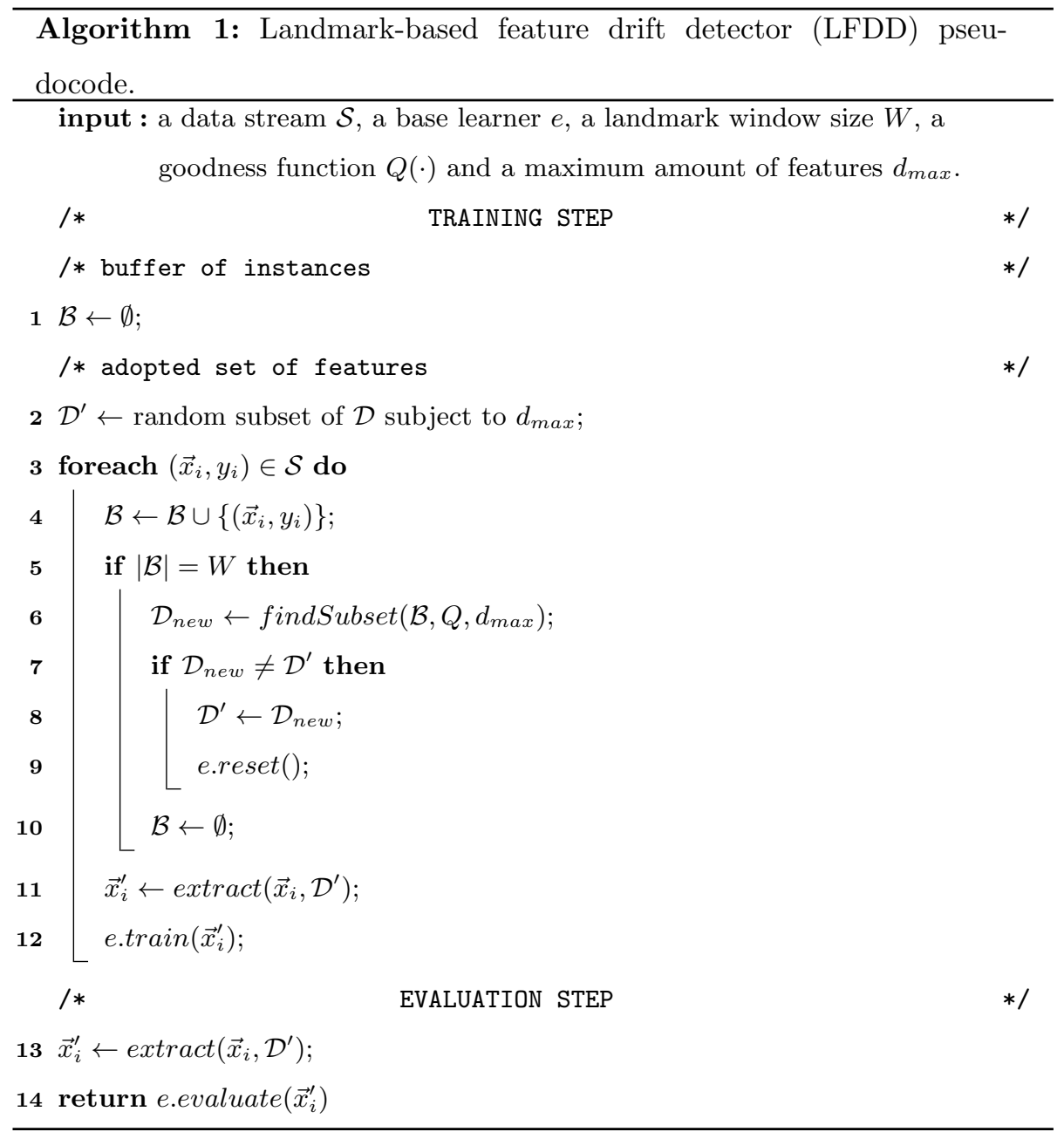

this section. In these plots, we mark with a dot the base learner behavior, i.e. the base learner behavior learning with all original features. Cases where no dots appear for a given learner indicate that LFDD outperformed the base learner in all cases, independently of $d_{\max }$.

In Fig. 6 it is possible to verify that LFDD is able to boost all base learners by adopting any goodness function and for a wide range of values of $d_{\max }$. For instance, a conventional NB, when combined to LFDD, is able to improve over its default setting by selecting between 2 and 42 features. In other cases, 
such as presented in Figs. $6 \mathrm{~b}$ and $6 \mathrm{c}$, it is possible to see that, Information Gain and Gain Ratio, when applied to both NB and VFDT are superior to the conventional base learner setting in all cases, i.e. when $d_{\max } \in[2 ; 49]$. This highlights that both the original VFDT and NB are incapable of quickly adapting to feature drifts and the usage of LFDD helps in feature drift detection and model adaptation.

In Figs. 8 and 9 we present the processing time results for the same experiments, where one can see that processing time increases according to the growth in the dimensionality adopted $d_{\max }$. First, it is important to notice that both NB and VFDT, when combined to LFDD, do not present significant improvements in processing time. This occurs due to the simple learning scheme adopted by both algorithms: the first (NB) works with a simple contingency table, which requires a $\mathcal{O}\left(d_{\max }\right)$ to classify each instance; while the second (VFDT) requires at maximum $\mathcal{O}\left(\log _{2} d_{\text {max }}\right)$. Although there is no interesting gain being achieved for processing time by adopting LFDD, we recall that there is a gain in accuracy (Figs. 6 and 7).

On the other hand, two other cases are the opposite: $1 N N$ and VFDR. To classify each instance a $1 N N$ classifier acts in $\mathcal{O}\left(N \times d_{\max }\right)$, therefore, the value of $d_{\max }$ highly impacts processing time. Conversely, VFDR exhibits an increased processing time due to the rule set, that grows according to $d_{\max }$. Again, we mark the base learner default behavior, showing that in most cases, working on a reduced dimensionality up to a given threshold results in less processing time for synthetic experiments. We highlight the Spam Corpus experiment, where are all base learners, when associated with LFDD and any heuristic goodness measures, resulted in less processing time when compared to their default configuration, i.e. no feature selection.

Figs. 10 and 11 present RAM-Hours results for SEA-FD(G) and Spam Corpus experiments where one can see that with the increase of $d_{\max }$, algorithms increase their memory usage as well. Again, we highlight both $1 N N$ and VFDR, since the first stores in memory a buffer with $\mathcal{O}\left(N \times d_{\max }\right)$ space and the second has its rule set growing exponentially with $d_{\max }$. 


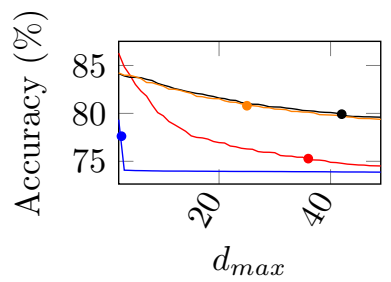

(a) Correlation

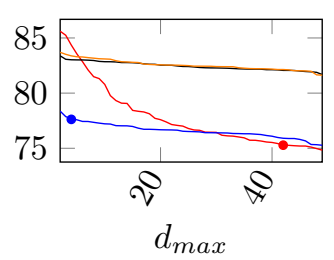

(b) Information Gain

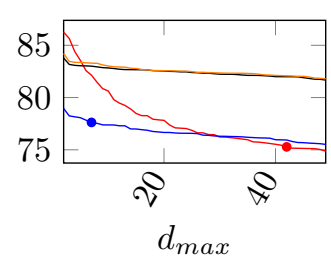

(c) Gain Ratio

\section{$-1 N N-\mathrm{NB}-\mathrm{VFDR}-\mathrm{VFDT}$}

Figure 6: Accuracy obtained by LFDD during the SEA-FD $(\mathrm{G})$ experiment.

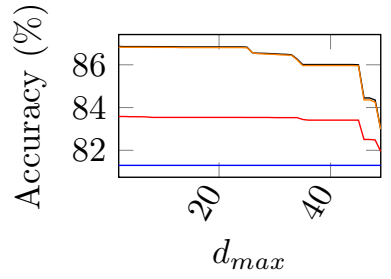

(a) Correlation

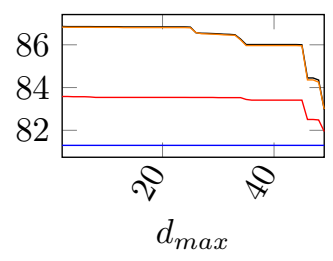

(b) Information Gain

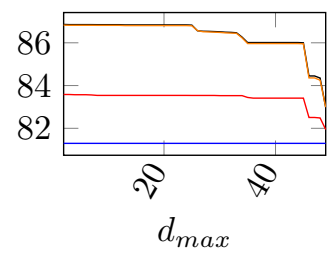

(c) Gain Ratio

$$
-1 N N-\mathrm{NB}-\mathrm{VFDR}-\mathrm{VFDT}
$$

Figure 7: Accuracy obtained by LFDD during the Spam Corpus experiment.

We emphasize that when comparing accuracy, processing time, and memory usage, both information gain and gain ratio have presented interesting results. While heuristic goodness functions were able to boost LFDD's base learner's accuracy, the overhead of selecting features and converting arriving instances into this reduced dimensionality also provided descreases in both processing time and memory space usage when compared to the default configuration of each base learner. This empirically shows that feature selection, even for data streams, is able to provide machine learning models with higher accuracy, for less processing time and memory space [11].

Finally, we present the results obtained by the adoption of LFDD in all experiments in accuracy (Tab. 5), processing time (Tab. 6) and memory space 


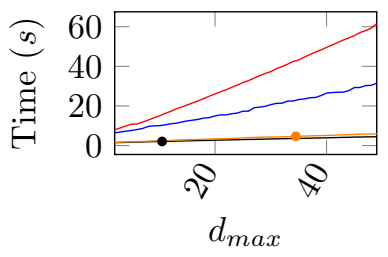

(a) Correlation

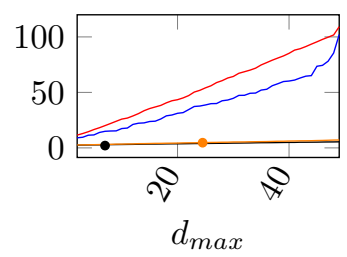

(b) Information Gain

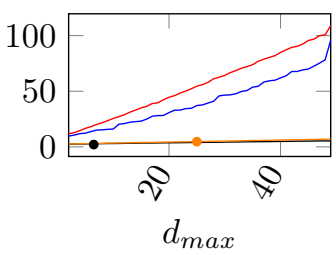

(c) Gain Ratio

$-1 N N-\mathrm{NB}-\mathrm{VFDR}-\mathrm{VFDT}$

Figure 8: Processing time $(s)$ obtained during the SEA-FD $(\mathrm{G})$ experiment.

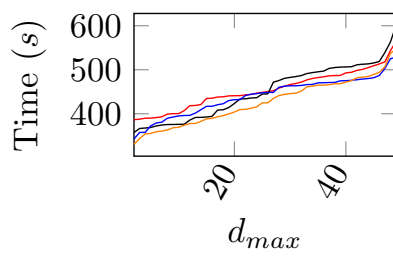

(a) Correlation

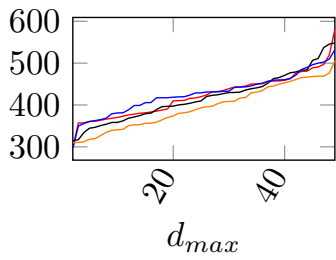

(b) Information Gain

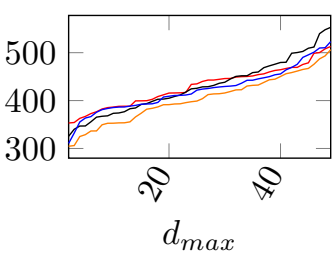

(c) Gain Ratio

$$
-1 N N-\mathrm{NB}-\mathrm{VFDR}-\mathrm{VFDT}
$$

Figure 9: Processing time $(s)$ obtained by LFDD during the Spam Corpus experiment.

(Tab. 7). Our intent was to perform a pessimistic evaluation of LFDD, therefore, the results presented in the latter cited Tables reflect the lowest accuracy obtained by LFDD regardless of the goodness function adopted, and highest processing time and memory usage. In order to assess whether the usage of LFDD presents significant differences when compared to an isolated base classifier, we performed several paired Wilcoxon's tests.

In terms of accuracy, Wilcoxon's test pointed out that LFDD outperforms base classifiers in all cases by assuming a $95 \%$ confidence level. This fact shows that a simple landmark-based filter is able to produce interesting feature drift adaptation when compared to conventional learners. It is also important to emphasize that both VFDT and VFDR had their results boosted, therefore 


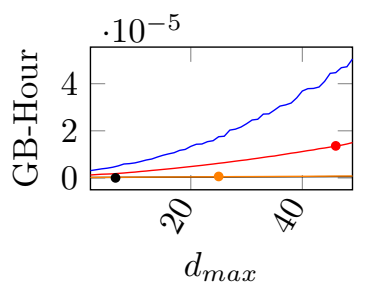

(a) Correlation

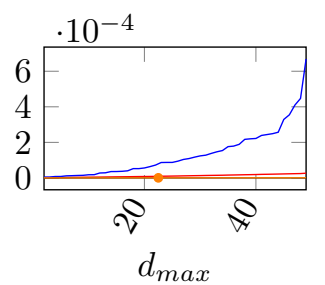

(b) Information Gain

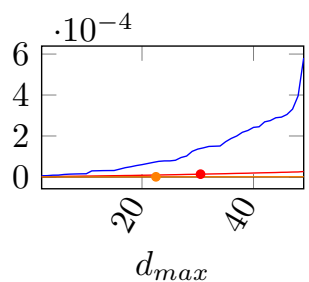

(c) Gain Ratio

$-1 N N-\mathrm{NB}-\mathrm{VFDR}-\mathrm{VFDT}$

Figure 10: RAM-Hours obtained during the SEA-FD $(\mathrm{G})$ experiment.

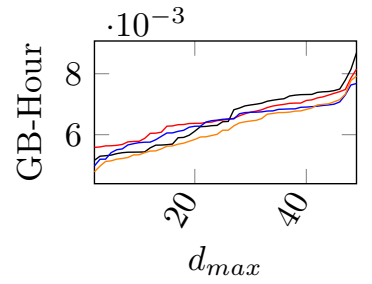

(a) Correlation

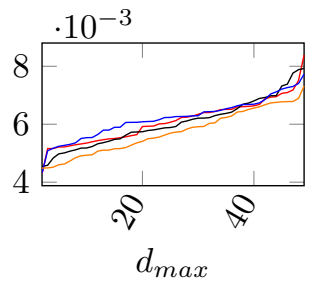

(b) Information Gain

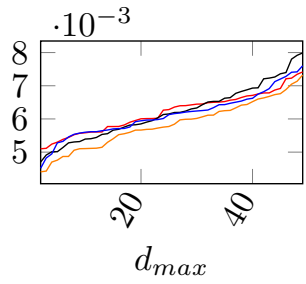

(c) Gain Ratio

\section{$1 N N-\mathrm{NB}-\mathrm{VFDR}-\mathrm{VFDT}$}

Figure 11: RAM-Hours obtained by LFDD during the Spam Corpus experiment.

highlighting the fact that their models are unable to adapt to feature drift more promptly.

In terms of processing time and memory usage, one can see that the adoption of LFDD when compared to the original learners, allows for faster computation and lower memory consumption in all cases, results corroborated by Wilcoxon's test. By combining results across all three aspects, one can see that performing periodical evaluations of features' discriminative power consistently leads to smaller subsets of features for classifiers to work with. As in batch learning, we showed that feature selection is beneficial since it allows learners, on average, to obtain higher accuracy, while reducing both processing time and memory usage. 
Table 5: Accuracy obtained by algorithms with and without LFDD.

\begin{tabular}{ccc|cc|cc|cc}
\hline \multicolumn{7}{c}{ Accuracy (\%) } \\
\hline Experiment & NB & LFDD-NB & VFDT & LFDD-VFDT & VFDR & LFDD-VFDR & $1 N N$ & LFDD-1NN \\
\hline RTG-FD(A) & 55.43 & $\mathbf{8 4 . 9 0}$ & 55.65 & $\mathbf{8 4 . 7 6}$ & $\mathbf{5 6 . 4 3}$ & 55.42 & $\mathbf{5 7 . 2 1}$ & 56.43 \\
RTG-FD(G) & 55.41 & $\mathbf{8 4 . 1 9}$ & 55.68 & $\mathbf{8 4 . 2 3}$ & $\mathbf{5 5 . 8 6}$ & 55.37 & $\mathbf{5 7 . 1 4}$ & 56.24 \\
SEA-FD(A) & 79.83 & $\mathbf{7 9 . 9 6}$ & 80.82 & $\mathbf{8 6 . 8 2}$ & $\mathbf{7 8 . 9 2}$ & 78.57 & 75.28 & $\mathbf{8 6 . 3 1}$ \\
SEA-FD(G) & 78.92 & $\mathbf{7 9 . 3 5}$ & 80.80 & $\mathbf{8 4 . 2 3}$ & 77.62 & $\mathbf{7 9 . 2 0}$ & 75.28 & $\mathbf{8 6 . 2 7}$ \\
BG1-FD(A) & 69.99 & $\mathbf{9 4 . 0 6}$ & 78.21 & $\mathbf{9 3 . 8 1}$ & 72.67 & $\mathbf{8 0 . 1 1}$ & 86.00 & $\mathbf{8 8 . 9 8}$ \\
BG1-FD(G) & 69.99 & $\mathbf{9 3 . 0 3}$ & 78.25 & $\mathbf{9 2 . 8 1}$ & 70.21 & $\mathbf{7 9 . 3 7}$ & 85.69 & $\mathbf{8 9 . 0 1}$ \\
BG2-FD(A) & 62.02 & $\mathbf{8 1 . 4 4}$ & 66.63 & $\mathbf{8 6 . 8 8}$ & 68.17 & $\mathbf{7 4 . 1 8}$ & 73.20 & $\mathbf{8 7 . 8 8}$ \\
BG2-FD(G) & 61.94 & $\mathbf{7 6 . 3 2}$ & 66.73 & $\mathbf{8 6 . 1 7}$ & 66.41 & $\mathbf{7 3 . 8 5}$ & 72.89 & $\mathbf{8 6 . 7 2}$ \\
BG3-FD(A) & 54.99 & $\mathbf{5 7 . 7 3}$ & 62.43 & $\mathbf{7 3 . 9 2}$ & 54.24 & $\mathbf{5 7 . 4 0}$ & 65.96 & $\mathbf{8 3 . 4 6}$ \\
BG3-FD(G) & 54.74 & $\mathbf{5 6 . 7 4}$ & 60.70 & $\mathbf{6 9 . 9 2}$ & 54.07 & $\mathbf{5 7 . 7 0}$ & 65.93 & $\mathbf{8 0 . 6 8}$ \\
Spam Corpus & 86.64 & $\mathbf{8 6 . 8 5}$ & 86.47 & $\mathbf{8 6 . 8 3}$ & 74.81 & $\mathbf{8 1 . 3 0}$ & 79.85 & $\mathbf{8 3 . 5 8}$ \\
\hline
\end{tabular}

\section{Research Challenges and Future Directions}

Determining the most discriminative subset of features as a data stream progresses is not straightforward. In this paper we presented and benchmarked existing works that perform feature drift adaptation in both explicit and implicit fashions. This survey shows that feature drift is another challenging trait of streaming scenarios that must be accounted for by new stream learning algorithms. Through our naive proposal, namely LFDD, we showed that it is possible to perform feature selection as the stream progresses and that this allows for quicker feature drift recovery and reduces overall processing time and memory usage. Nevertheless, there exists a number of research questions that are still unanswered and pose challenges for the streaming research community.

Inductive tree learning is one of the most commonly used approaches for classifying data streams. As discussed in Sec. 4, very few decision trees regard the possibility of changes in the underlying distribution of data, and therefore introduce some kind of pruning strategy into the tree evolution. With rare exceptions, existing strategies are based on equal-sized windowing techniques, where the algorithm verifies if the attributes used in split nodes are still maximizing a goodness function $Q(\cdot)$, or if they should be replaced by more appropriate splits. 
Table 6: Processing time obtained by algorithms with and without LFDD.

\begin{tabular}{ccc|cc|cc|cc}
\hline \multicolumn{7}{c}{ Processing time $(s)$} \\
\hline Experiment & NB & LFDD-NB & VFDT & LFDD-VFDT & VFDR & LFDD-VFDR & $1 N N$ & LFDD-1NN \\
\hline RTG-FD(A) & 1.94 & $\mathbf{1 . 7 7}$ & 6.66 & $\mathbf{6 . 5 3}$ & 85.21 & $\mathbf{8 0 . 9 5}$ & 7.49 & $\mathbf{6 . 5 9}$ \\
RTG-FD(G) & 1.62 & $\mathbf{1 . 4 7}$ & 6.07 & $\mathbf{5 . 7 7}$ & 94.20 & $\mathbf{8 5 . 7 2}$ & 7.59 & $\mathbf{6 . 5 3}$ \\
SEA-FD(A) & $\mathbf{1 . 9 3}$ & 2.07 & 4.36 & $\mathbf{4 . 2 3}$ & 424.62 & $\mathbf{3 9 9 . 1 4}$ & 110.43 & $\mathbf{7 . 8 4}$ \\
SEA-FD(G) & $\mathbf{1 . 9 7}$ & 2.01 & 4.68 & $\mathbf{4 . 4 9}$ & 192.48 & $\mathbf{1 8 2 . 8 6}$ & 110.38 & $\mathbf{7 . 9 5}$ \\
BG1-FD(A) & 1.45 & $\mathbf{1 . 3 1}$ & 4.61 & $\mathbf{4 . 5 2}$ & 5.50 & $\mathbf{5 . 0 0}$ & 2.66 & $\mathbf{2 . 2 6}$ \\
BG1-FD(G) & 1.43 & $\mathbf{1 . 3 2}$ & 4.44 & $\mathbf{4 . 2 6}$ & 5.54 & $\mathbf{5 . 2 1}$ & 2.86 & $\mathbf{2 . 5 2}$ \\
BG2-FD(A) & 1.59 & $\mathbf{1 . 5 1}$ & 4.04 & $\mathbf{4 . 0 0}$ & 7.55 & $\mathbf{6 . 8 7}$ & 3.18 & $\mathbf{2 . 8 6}$ \\
BG2-FD(G) & 1.37 & $\mathbf{1 . 2 9}$ & 3.98 & $\mathbf{3 . 8 6}$ & 6.57 & $\mathbf{6 . 1 8}$ & 3.84 & $\mathbf{3 . 4 2}$ \\
BG3-FD(A) & 1.46 & $\mathbf{1 . 3 6}$ & 3.37 & $\mathbf{3 . 2 4}$ & 5.35 & $\mathbf{5 . 0 8}$ & 3.40 & $\mathbf{2 . 9 2}$ \\
BG3-FD(G) & 1.33 & $\mathbf{1 . 2 1}$ & 3.67 & $\mathbf{3 . 6 3}$ & 5.75 & $\mathbf{5 . 2 3}$ & 4.09 & $\mathbf{3 . 4 8}$ \\
Spam Corpus & 617.66 & $\mathbf{5 8 6 . 7 8}$ & 695.06 & $\mathbf{6 6 7 . 2 6}$ & 691.51 & $\mathbf{6 3 6 . 1 9}$ & 6329.34 & $\mathbf{5 5 0 6 . 5 3}$ \\
\hline
\end{tabular}

Table 7: RAM-Hours obtained by algorithms with and without LFDD.

\begin{tabular}{|c|c|c|c|c|c|c|c|c|}
\hline \multicolumn{9}{|c|}{ RAM-Hours (GB-Hour) } \\
\hline Experiment & NB & LFDD-NB & VFDT & LFDD-VFDT & VFDR & LFDD-VFDR & $1 N N$ & LFDD- $1 N N$ \\
\hline RTG-FD(A) & $1.76 \times 10^{-8}$ & $1.64 \times 10^{-8}$ & $1.85 \times 10^{-6}$ & $1.74 \times 10^{-6}$ & $4.64 \times 10^{-4}$ & $4.59 \times 10^{-4}$ & $1.18 \times 10^{-6}$ & $1.16 \times 10^{-6}$ \\
\hline RTG-FD(G) & $1.62 \times 10^{-8}$ & $1.51 \times 10^{-8}$ & $1.81 \times 10^{-6}$ & $1.68 \times 10^{-6}$ & $4.91 \times 10^{-4}$ & $4.86 \times 10^{-4}$ & $1.21 \times 10^{-6}$ & $1.19 \times 10^{-6}$ \\
\hline SEA-FD(A) & $1.56 \times 10^{-8}$ & $1.47 \times 10^{-8}$ & $6.08 \times 10^{-7}$ & $5.72 \times 10^{-7}$ & $1.04 \times 10^{-2}$ & $1.02 \times 10^{-2}$ & $1.36 \times 10^{-5}$ & $1.35 \times 10^{-5}$ \\
\hline SEA-FD(G) & $1.42 \times 10^{-8}$ & $1.32 \times 10^{-8}$ & $6.20 \times 10^{-7}$ & $5.83 \times 10^{-7}$ & $2.32 \times 10^{-3}$ & $2.23 \times 10^{-3}$ & $1.36 \times 10^{-5}$ & $1.35 \times 10^{-5}$ \\
\hline BG1-FD(A) & $1.21 \times 10^{-8}$ & $1.13 \times 10^{-8}$ & $7.36 \times 10^{-7}$ & $6.84 \times 10^{-7}$ & $2.95 \times 10^{-7}$ & $2.80 \times 10^{-7}$ & $3.99 \times 10^{-8}$ & $3.91 \times 10^{-8}$ \\
\hline BG1-FD(G) & $1.29 \times 10^{-8}$ & $1.20 \times 10^{-8}$ & $7.25 \times 10^{-7}$ & $6.74 \times 10^{-7}$ & $3.15 \times 10^{-7}$ & $3.09 \times 10^{-7}$ & $4.71 \times 10^{-8}$ & $4.62 \times 10^{-8}$ \\
\hline BG2-FD(A) & $1.32 \times 10^{-8}$ & $1.23 \times 10^{-8}$ & $4.91 \times 10^{-7}$ & $4.62 \times 10^{-7}$ & $6.34 \times 10^{-7}$ & $6.28 \times 10^{-7}$ & $6.70 \times 10^{-8}$ & $6.63 \times 10^{-8}$ \\
\hline BG2-FD(G) & $1.23 \times 10^{-8}$ & $1.16 \times 10^{-8}$ & $4.45 \times 10^{-7}$ & $4.14 \times 10^{-7}$ & $4.97 \times 10^{-7}$ & $4.92 \times 10^{-7}$ & $8.04 \times 10^{-8}$ & $7.88 \times 10^{-8}$ \\
\hline BG3-FD(A) & $1.32 \times 10^{-8}$ & $1.24 \times 10^{-8}$ & $2.89 \times 10^{-7}$ & $2.69 \times 10^{-7}$ & $2.54 \times 10^{-7}$ & $2.51 \times 10^{-7}$ & $7.57 \times 10^{-8}$ & $7.42 \times 10^{-8}$ \\
\hline BG3-FD(G) & $1.20 \times 10^{-8}$ & $1.13 \times 10^{-8}$ & $3.65 \times 10^{-7}$ & $3.43 \times 10^{-7}$ & $2.99 \times 10^{-7}$ & $2.84 \times 10^{-7}$ & $1.19 \times 10^{-7}$ & $1.17 \times 10^{-7}$ \\
\hline Spam Corpus & $2.34 \times 10^{-2}$ & $2.18 \times 10^{-2}$ & $1.56 \times 10^{-2}$ & $1.45 \times 10^{-2}$ & $5.11 \times 10^{-2}$ & $4.96 \times 10^{-2}$ & $5.50 \times 10^{-2}$ & $5.45 \times 10^{-2}$ \\
\hline
\end{tabular}

The same can be said for decision rule learning. Algorithms like Facil and VFDR do not encompass strategies for adapting its model to drifts in data, therefore they must be accompanied by drift detectors (e.g. ADWIN [50] and Page-Hinkley's test [68]) that periodically reset the entire rule set according to error rates of the classifier.

Through randomness and combinatorics, the latter approaches can be combined into ensembles to boost accuracy and to allow for implicit drift adaptation. Nevertheless, training and maintaining an ensemble is not only computationally costly, but it must also employ specific diversity induction and voting schemes.

By randomness, Streaming Random Forests and Random Rules create en- 
sembles and each of its experts are associated with a random subset of features $\mathcal{D}^{\prime}$. Arriving instances are then used to train experts after their conversion to $\mathcal{D}^{\prime}$. Due to randomness, it is necessary that experts are allocated with $\mathcal{D}^{\prime}$ that cover diverse areas of the feature subsets space. The assumption is that at least one of the experts is associated with a useful $\mathcal{D}^{\prime} \supseteq \mathcal{D}^{*}$. By associating each expert with a dynamic weight that grows and shrinks accordingly to correct and misclassified instances, the ensemble implicitly adapts to feature drifts since experts with the most discriminative subsets will present higher accuracy rates.

Analogously, the same algorithms can form ensembles by exploring combinatorics. Assuming a feature set $\mathcal{D}$ with $|\mathcal{D}|=M$, it is necessary to create an ensemble with $\sum_{i=1}^{M}\left(\begin{array}{c}M \\ i\end{array}\right)$ experts. Again, by associating each expert with a subset $\mathcal{D}^{\prime}$ and a dynamic weight, the one with $\mathcal{D}^{\prime}=\mathcal{D}^{*}$ will present higher accuracy rates and will outvote other experts in predictions. Nevertheless, by exploring combinatorics the size of the ensemble becomes intractable as the size of the experts grows very quickly with $M$.

Finally, approaches like CVFDT [45], HEFT-Stream [21] and LFDD assume that the most discriminative subset of features can be computed by filters on disjoint chunks of instances. These algorithms have outperformed others in experiments, however, their major limitation is how to determine the size of these windows, which directly affects the learning process. Small windows allow for quicker recognition of possible changes in the chosen subset of features, however, this approach may lead to the detection of false changes if the stream is noisy. Conversely, bigger windows enable a larger amount of data to work on, yet fail to quickly detect changes in the most discriminative subset.

Another open question regards how each classifier deals with changes in this chosen discriminative subset. For example, if a change is detected in a decision tree or decision rule learning algorithm, it is possible to adapt the model learned in order to avoid full model reset, e.g. Hoeffding Adaptive Tree [46], however, the same might not hold for other types of learners.

Therefore, open research topics include the development of techniques that constantly verify the relevance of features as new instances arrive in an adaptive 
and incremental fashion. Performing such verification as data arrives, and independently of window sizes and base classifiers is important, since it allows for faster recognition of feature drifts and improves a classifier's overall accuracy and processing time.

\section{Conclusion}

This paper presented, formalized and exemplified one rarely addressed characteristic of data streams: feature drifts. Additionally, we surveyed and benchmarked algorithms that perform feature selection during stream learning in both explicit and implicit fashions. Results obtained highlight that feature drift is another challenging trait of data streams that must be accounted for by new stream learning algorithms.

Besides serving as an introduction into the research area of dynamic feature selection for data streams, we expect that this paper helps to position new adaptive learning techniques and applications to which these apply.

As a conclusion, we believe that performing dynamic feature selection in data streams has not received proper attention in the current research scenario. Studying how to perform dynamic feature selection as streams progress enables algorithms to work only with the most relevant features by discarding irrelevant ones. Throughout simple experiments based on a naive proposal, we showed that a classifier's accuracy can be boosted in feature drifting data, while reducing both processing time and memory space. We hope that the results presented here will motivate more research into developing incremental and adaptive feature selection for data streams.

\section{References}

[1] J. P. Barddal, H. M. Gomes, F. Enembreck, Sfnclassifier: A scale-free social network method to handle concept drift, in: Proceedings of the 29th Annual ACM Symposium on Applied Computing, SAC '14, ACM, New York, NY, 
USA, 2014, pp. 786-791. doi:10.1145/2554850.2554855.

URL http://doi.acm.org/10.1145/2554850.2554855

[2] A. Bifet, J. Read, I. Zliobaite, B. Pfahringer, G. Holmes, Pitfalls in benchmarking data stream classification and how to avoid them, in: ECML/PKDD (1), 2013, pp. 465-479.

[3] J. Gama, P. Kosina, Learning decision rules from data streams, in: IJCAI 2011, Proceedings of the 22nd International Joint Conference on Artificial Intelligence, Barcelona, Catalonia, Spain, July 16-22, 2011, 2011, pp. 12551260.

[4] C. C. Aggarwal, J. Han, J. Wang, P. S. Yu, A framework for clustering evolving data streams, in: Proceedings of the 29th International Conference on Very Large Data Bases - Volume 29, VLDB '03, VLDB Endowment, 2003, pp. 81-92.

[5] J. P. Barddal, H. M. Gomes, F. Enembreck, Sncstream: A social networkbased data stream clustering algorithm, in: Proceedings of the 30th Annual ACM Symposium on Applied Computing, SAC '15, ACM, New York, NY, USA, 2015, pp. 935-940. doi:10.1145/2695664.2695674.

URL http://doi.acm.org/10.1145/2695664.2695674

[6] F. Cao, M. Ester, W. Qian, A. Zhou, Density-based clustering over an evolving data stream with noise, in: SDM, 2006, pp. 328-339.

[7] P. Kranen, I. Assent, C. Baldauf, T. Seidl, The clustree: Indexing microclusters for anytime stream mining, Knowl. Inf. Syst. 29 (2) (2011) 249-272. doi:10.1007/s10115-010-0342-8.

[8] J. a. Gama, I. Zliobaite, A. Bifet, M. Pechenizkiy, A. Bouchachia, A survey on concept drift adaptation, ACM Comput. Surv. 46 (4) (2014) 44:1-44:37. doi:10.1145/2523813. 
[9] A. Tsymbal, The problem of concept drift: definitions and related work, Tech. Rep. TCD-CS-2004-15, The University of Dublin, Trinity College, Department of Computer Science, Dublin, Ireland (2004).

[10] J. P. Barddal, H. M. Gomes, F. Enembreck, A survey on feature drift adaptation, in: Tools with Artificial Intelligence (ICTAI), 2015 IEEE 27th International Conference on, 2015, pp. 1053-1060. doi:10.1109/ICTAI.2015.150.

[11] K. Naidu, A. Dhenge, K. Wankhade, Feature selection algorithm for improving the performance of classification: A survey, in: Proceedings of the 2014 Fourth International Conference on Communication Systems and Network Technologies, CSNT '14, IEEE Computer Society, Washington, DC, USA, 2014, pp. 468-471. doi:10.1109/CSNT.2014.99.

[12] A. Amini, T. Y. Wah, On density-based data streams clustering algorithms: A survey, Journal of Computer Science and Technology 29 (1) (2014) 116141. doi:10.1007/s11390-014-1416-y.

[13] A. Bifet, B. Pfahringer, J. Read, G. Holmes, Efficient data stream classification via probabilistic adaptive windows, in: SAC, 2013, pp. 801-806.

[14] A. Bifet, Adaptive Stream Mining: Pattern Learning and Mining from Evolving Data Streams, Vol. 207 of Frontiers in Artificial Intelligence and Applications, IOS Press, 2010.

[15] E. Almeida, P. Kosina, J. Gama, Random rules from data streams, in: Proceedings of the 28th Annual ACM Symposium on Applied Computing, SAC '13, Coimbra, Portugal, March 18-22, 2013, 2013, pp. 813-814. doi:10.1145/2480362.2480518.

[16] P. Kosina, J. Gama, Very fast decision rules for multi-class problems, in: Proceedings of the 27th Annual ACM Symposium on Applied Computing, SAC '12, ACM, New York, NY, USA, 2012, pp. 795-800. doi:10.1145/2245276.2245431. 
[17] C. C. Aggarwal, A. Hinneburg, D. A. Keim, On the surprising behavior of distance metrics in high dimensional space, in: J. Van den Bussche, V. Vianu (Eds.), Database Theory ICDT 2001, Vol. 1973 of Lecture Notes in Computer Science, Springer Berlin Heidelberg, 2001, pp. 420-434. doi:1.1007/3-540-44503-X_27.

[18] J. Gama, Knowledge Discovery from Data Streams, 1st Edition, Chapman \& Hall/CRC, 2010.

[19] H. Abdulsalam, D. B. Skillicorn, P. Martin, Classification using streaming random forests, IEEE Trans. on Knowl. and Data Eng. 23 (1) (2011) 22-36. doi:10.1109/TKDE.2010.36.

[20] J. A. Silva, E. R. Faria, R. C. Barros, E. R. Hruschka, A. C. P. L. F. d. Carvalho, J. a. Gama, Data stream clustering: A survey, ACM Comput. Surv. 46 (1) (2013) 13:1-13:31. doi:10.1145/2522968.2522981.

[21] H.-L. Nguyen, Y.-K. Woon, W.-K. Ng, L. Wan, Heterogeneous ensemble for feature drifts in data streams, in: P.-N. Tan, S. Chawla, C. Ho, J. Bailey (Eds.), Advances in Knowledge Discovery and Data Mining, Vol. 7302 of Lecture Notes in Computer Science, Springer Berlin Heidelberg, 2012, pp. 1-12. doi:10.1007/978-3-642-30220-6_1.

[22] P. Domingos, A few useful things to know about machine learning, Commun. ACM 55 (10) (2012) 78-87. doi:10.1145/2347736.2347755.

[23] S. C. H. Hoi, J. Wang, P. Zhao, R. Jin, Online feature selection for mining big data, in: Proceedings of the 1st International Workshop on Big Data, Streams and Heterogeneous Source Mining: Algorithms, Systems, Programming Models and Applications, BigMine '12, ACM, New York, NY, USA, 2012, pp. 93-100. doi:10.1145/2351316.2351329.

[24] H.-G. Li, X. Wu, Z. Li, W. Ding, Online group feature selection from feature streams, in: M. des Jardins, M. L. Littman (Eds.), AAAI, AAAI Press, 2013, pp. 1627-1628. 
[25] R. Kohavi, G. H. John, Wrappers for feature subset selection, Artificial Intelligence 97 (12) (1997) 273 - 324, relevance. doi:http://dx.doi.org/10.1016/S0004-3702(97)00043-X.

[26] W. R. Rudnicki, M. Wrzesien, W. Paja, All relevant feature selection methods and applications, in: U. Stańczyk, L. C. Jain (Eds.), Feature Selection for Data and Pattern Recognition, Vol. 584 of Studies in Computational Intelligence, Springer Berlin Heidelberg, 2015, pp. 11-28. doi:10.1007/9783-662-45620-0_2.

[27] Z. Zhao, F. Morstatter, S. Sharma, S. Alelyani, A. Anand, H. Liu, Advancing feature selection research, ASU feature selection repository (2010) $1-28$.

[28] L. Yu, H. Liu, Feature selection for high-dimensional data: A fast correlation-based filter solution, in: Proceedings of the Twentieth International Conference on Machine Learning, AAAI Press, 2003, pp. 856-863.

[29] R. Duangsoithong, T. Windeatt, Relevant and redundant feature analysis with ensemble classification, in: Advances in Pattern Recognition, 2009. ICAPR '09. Seventh International Conference on, 2009, pp. 247-250. doi:10.1109/ICAPR.2009.36.

[30] M. A. Hall, L. A. Smith, Feature selection for machine learning: Comparing a correlation-based filter approach to the wrapper, in: Proceedings of the Twelfth International Florida Artificial Intelligence Research Society Conference, AAAI Press, 1999, pp. 235-239.

URL http://dl.acm.org/citation.cfm?id=646812.707499

[31] M. A. Hall, Correlation-based feature selection for discrete and numeric class machine learning, in: Proceedings of the Seventeenth International Conference on Machine Learning, ICML '00, Morgan Kaufmann Publishers Inc., San Francisco, CA, USA, 2000, pp. 359-366. 
[32] T. F. Covões, E. R. Hruschka, L. N. de Castro, A. M. Santos, A clusterbased feature selection approach, in: E. Corchado, X. Wu, E. Oja, A. Herrero, B. Baruque (Eds.), Hybrid Artificial Intelligence Systems, Vol. 5572 of Lecture Notes in Computer Science, Springer Berlin Heidelberg, 2009, pp. 169-176. doi:10.1007/978-3-642-02319-4_20.

[33] C. H. Park, A feature selection method using hierarchical clustering, in: R. Prasath, T. Kathirvalavakumar (Eds.), Mining Intelligence and Knowledge Exploration, Vol. 8284 of Lecture Notes in Computer Science, Springer International Publishing, 2013, pp. 1-6. doi:10.1007/978-3-319-03844-5_1.

[34] V. R. Carvalho, W. W. Cohen, Single-pass online learning: Performance, voting schemes and online feature selection, in: Proceedings of the 12th ACM SIGKDD International Conference on Knowledge Discovery and Data Mining, KDD '06, ACM, New York, NY, USA, 2006, pp. 548-553. doi:10.1145/1150402.1150466.

[35] I. Guyon, An introduction to variable and feature selection, Journal of Machine Learning Research 3 (2003) 1157-1182.

[36] C. C. Aggarwal, An introduction to data classification, in: Data Classification: Algorithms and Applications, CRC Press, Taylor \& Francis Group, 2014, pp. 1-36.

[37] P. Domingos, G. Hulten, Mining high-speed data streams, in: Proceedings of the Sixth ACM SIGKDD International Conference on Knowledge Discovery and Data Mining, KDD '00, ACM, New York, NY, USA, 2000, pp. 71-80. doi:10.1145/347090.347107.

[38] J. P. Barddal, H. M. Gomes, F. Enembreck, Neural Information Processing: 22nd International Conference, ICONIP 2015, Istanbul, Turkey, November 9-12, 2015, Proceedings, Part I, Springer International Publishing, Cham, 2015, Ch. Analyzing the Impact of Feature Drifts in Streaming Learning, pp. 21-28. doi:10.1007/978-3-319-26532-2_3. 
[39] I. Katakis, G. Tsoumakas, I. Vlahavas, Dynamic feature space and incremental feature selection for the classification of textual data streams, in: in ECML/PKDD-2006 International Workshop on Knowledge Discovery from Data Streams. 2006, Springer Verlag, 2006, p. 107.

[40] M. Hall, E. Frank, G. Holmes, B. Pfahringer, P. Reutemann, I. H. Witten, The weka data mining software: An update, SIGKDD Explor. Newsl. 11 (1) (2009) 10-18. doi:10.1145/1656274.1656278.

[41] J. Zhou, D. Foster, R. Stine, L. Ungar, Streaming feature selection using alpha-investing, in: Proceedings of the Eleventh ACM SIGKDD International Conference on Knowledge Discovery in Data Mining, KDD '05, ACM, New York, NY, USA, 2005, pp. 384-393. doi:10.1145/1081870.1081914.

[42] F. J. Ferrer-Troyano, J. S. Aguilar-Ruiz, J. C. R. Santos, Incremental rule learning and border examples selection from numerical data streams, J. UCS 11 (8) (2005) 1426-1439. doi:10.3217/jucs-011-08-1426.

[43] H. Abdulsalam, D. Skillicorn, P. Martin, Streaming random forests, in: Database Engineering and Applications Symposium, 2007. IDEAS 2007. 11th International, 2007, pp. 225-232. doi:10.1109/IDEAS.2007.4318108.

[44] A. Bifet, E. Frank, G. Holmes, B. Pfahringer, Accurate ensembles for data streams: Combining restricted hoeffding trees using stacking, in: Proceedings of the 2nd Asian Conference on Machine Learning, ACML 2010, Tokyo, Japan, November 8-10, 2010, Vol. 13 of JMLR Proceedings, JMLR.org, 2010, pp. 225-240.

[45] G. Hulten, L. Spencer, P. Domingos, Mining time-changing data streams, in: Proceedings of the Seventh ACM SIGKDD International Conference on Knowledge Discovery and Data Mining, KDD '01, ACM, New York, NY, USA, 2001, pp. 97-106. doi:10.1145/502512.502529.

[46] A. Bifet, R. Gavaldà, Advances in Intelligent Data Analysis VIII: 8th International Symposium on Intelligent Data Analysis, IDA 2009, Lyon, 
France, August 31 - September 2, 2009. Proceedings, Springer Berlin Heidelberg, Berlin, Heidelberg, 2009, Ch. Adaptive Learning from Evolving Data Streams, pp. 249-260. doi:10.1007/978-3-642-03915-7_22.

[47] T. R. Hoens, N. V. Chawla, R. Polikar, Heuristic updatable weighted random subspaces for non-stationary environments, in: Data Mining (ICDM), 2011 IEEE 11th International Conference on, 2011, pp. 241-250. doi:10.1109/ICDM.2011.75.

[48] J. Han, M. Kamber, J. Pei, Data Mining: Concepts and Techniques, 3rd Edition, Morgan Kaufmann Publishers Inc., San Francisco, CA, USA, 2011.

[49] W. Hoeffding, Probability inequalities for sums of bounded random variables, Journal of the American Statistical Association 58 (301) (1963) 1330.

[50] A. Bifet, R. Gavald, Learning from time-changing data with adaptive windowing, in: Proceedings of the 2007 SIAM International Conference on Data Mining, 2007, pp. 443-448. doi:10.1137/1.9781611972771.42.

[51] A. Bifet, G. Holmes, B. Pfahringer, R. Gavaldà, Improving adaptive bagging methods for evolving data streams, in: Z.-H. Zhou, T. Washio (Eds.), Advances in Machine Learning, Vol. 5828 of Lecture Notes in Computer Science, Springer Berlin Heidelberg, 2009, pp. 23-37. doi:10.1007/978-3642-05224-8_4.

[52] A. Bifet, G. Holmes, B. Pfahringer, Leveraging bagging for evolving data streams, in: J. L. Balczar, F. Bonchi, A. Gionis, M. Sebag (Eds.), Machine Learning and Knowledge Discovery in Databases, Vol. 6321 of Lecture Notes in Computer Science, Springer Berlin Heidelberg, 2010, pp. 135-150. doi:10.1007/978-3-642-15880-3_15.

[53] N. Oza, Online bagging and boosting, in: Systems, Man and Cybernetics, 2005 IEEE International Conference on, Vol. 3, 2005, pp. 2340-2345 Vol. 3. doi:10.1109/ICSMC.2005.1571498. 
[54] T. G. Dietterich, Ensemble methods in machine learning, in: Proceedings of the First International Workshop on Multiple Classifier Systems, MCS '00, Springer-Verlag, London, UK, UK, 2000, pp. 1-15.

URL http://dl.acm.org/citation.cfm?id=648054.743935

[55] L. I. Kuncheva, W. J. Faithfull, PCA feature extraction for change detection in multidimensional unlabelled data, IEEE Transactions on Neural Networks and Learning Systems 25 (1) (2014) 69-80. doi:10.1109/TNNLS.2013.2248094.

[56] L. Breiman, Bagging predictors, Mach. Learn. 24 (2) (1996) 123-140. doi:10.1023/A:1018054314350.

[57] L. Breiman, Random forests, Machine Learning 45 (1) (2001) 5-32. doi:10.1023/A:1010933404324.

[58] J. Z. Kolter, M. A. Maloof, Dynamic weighted majority: An ensemble method for drifting concepts, J. Mach. Learn. Res. 8 (2007) 2755-2790.

[59] D. H. Wolpert, Stacked generalization, Neural Networks 5 (2) (1992) 241259. doi:10.1016/S0893-6080(05)80023-1.

[60] D. T. J. Huang, Y. S. Koh, G. Dobbie, A. Bifet, Machine Learning and Knowledge Discovery in Databases: European Conference, ECML PKDD 2015, Porto, Portugal, September 7-11, 2015, Proceedings, Part I, Springer International Publishing, 2015, Ch. Drift Detection Using Stream Volatility, pp. 417-432. doi:10.1007/978-3-319-23528-8_26.

[61] W. N. Street, Y. Kim, A streaming ensemble algorithm (sea) for largeclassification, in: Proc. of the seventh ACM SIGKDD international conference on Knowledge discovery and data mining, ACM SIGKDD, 2001, pp. 377-382.

[62] A. Bifet, G. Holmes, R. Kirkby, B. Pfahringer, MOA: Massive online analysis, The Journal of Machine Learning Research 11 (2010) 1601-1604. 
[63] J. Gama, P. Rodrigues, Issues in evaluation of stream learning algorithms, in: Proc. of the 15th ACM SIGKDD international conference on Knowledge discovery and data mining, ACM SIGKDD, 2009, pp. 329-338.

[64] F. Wilcoxon, Individual Comparisons by Ranking Methods, Biometrics Bulletin 1 (6) (1945) 80-83. doi:10.2307/3001968.

[65] M. Friedman, The Use of Ranks to Avoid the Assumption of Normality Implicit in the Analysis of Variance, Journal of the American Statistical Association 32 (200) (1937) 675-701. doi:10.2307/2279372.

[66] P. Nemenyi, Distribution-free multiple comparisons, Ph.D. thesis, New Jersey, USA (1963).

[67] K. Kira, L. A. Rendell, The feature selection problem: Traditional methods and a new algorithm, in: Proceedings of the Tenth National Conference on Artificial Intelligence, AAAI'92, AAAI Press, 1992, pp. 129-134.

[68] H. Mouss, D. Mouss, N. Mouss, L. Sefouhi, Test of page-hinckley, an approach for fault detection in an agro-alimentary production system, in: Control Conference, 2004. 5th Asian, Vol. 2, 2004, pp. 815-818 Vol.2. 